\title{
PERTURBATION BASED DECOMPOSITION OF SEMG SIGNALS
}

\author{
A Thesis \\ presented to \\ the Faculty of California Polytechnic State University,
}

San Luis Obispo

\author{
In Partial Fulfillment \\ of the Requirements for the Degree \\ Master of Science in Biomedical Engineering
}

by

Rachel Huettinger

March 2019 
(C) 2019

Rachel Huettinger

ALL RIGHTS RESERVED 


\title{
COMMITTEE MEMBERSHIP
}

\author{
TITLE: Perturbation Based Decomposition of \\ sEMG Signals
}

AUTHOR: Rachel Huettinger

DATE SUBMITTED: March 2019

COMMITTEE CHAIR: Robert Szlavik, Ph.D.

Professor of Biomedical Engineering

COMMITTEE MEMBER: David Clague, PhD

Professor of Biomedical Engineering

COMMITTEE MEMBER: Michael Whitt, PhD

Professor of Biomedical Engineering 


\section{Abstract \\ Perturbation Based Decomposition of sEMG Signals}

\section{Rachel Huettinger}

Surface electromyography records the motor unit action potential signals in the vicinity of the electrode to reveal information on muscle activation. Decomposition of sEMG signals for characterization of constituent motor unit action potentials in terms of amplitude and firing times is useful for clinical research as well as diagnosis of neurological disorders. Successful decomposition of sEMG signals would allow for pertinent motor unit action potential information to be acquired without discomfort to the subject or the need for a well-trained operator (compared with intramuscular EMG). To determine amplitudes and firing times for motor unit action potentials in an sEMG recording, Szlavik's perturbation based decomposition may be applied. The decomposition was initially applied to synthetic sEMG signals and then to experimental data collected from the biceps brachii. Szlavik's decomposition estimator yields satisfactory results for synthetic and experimental sEMG signals with reasonable complexity. 


\section{ACKNOWLEDGMENTS}

Thanks to:

- Dr. Szlavik

- Dr. Clague and Dr. Whitt for being on my committee

- My parents, Cathy and Curt, for their love and support

- EMGLAB for provision of experimental data [1]

- Andrew Guenther, for uploading this template [2] 


\section{TABLE OF CONTENTS}

Page

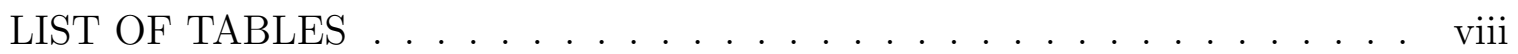

LIST OF FIGURES .............................. ix

\section{CHAPTER}

1 Introduction . . . . . . . . . . . . . . . . . 1

1.1 Motivation . . . . . . . . . . . . . . . . 1

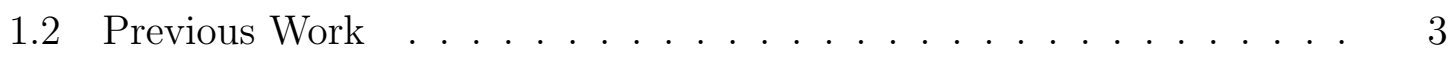

1.3 EMG Decomposition Challenges . . . . . . . . . . . . . . 4

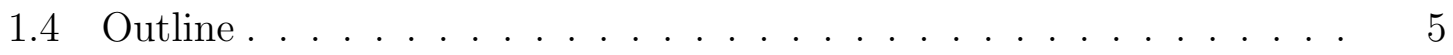

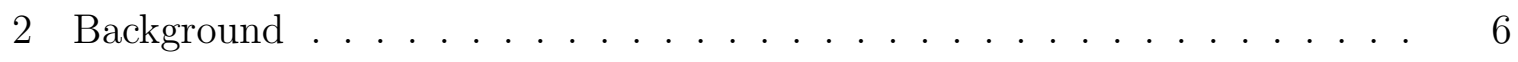

2.1 The Human Neuromuscular System . . . . . . . . . . . . . . 6

2.1.1 The Nervous System . . . . . . . . . . . . . 6

2.1.2 The Muscular System . . . . . . . . . . . . 6

2.1.3 The Neuromuscular Junction . . . . . . . . . . . . 8

2.2 Skeletal Muscle Activation . . . . . . . . . . . . . . . 9

2.2.1 Physiology of Muscle Fiber Activation . . . . . . . . . . . 9

2.2.2 Muscle Fiber Action Potential (MFAP) . . . . . . . . . . . . . 10

2.2.3 Motor Unit Action Potential (MUAP) . . . . . . . . . . . . 10

2.2.4 Motor Unit Action Potential Train (MUAPT) . . . . . . . . . 12

2.3 Electromyography Signal and Recording Techniques . . . . . . . . . . 12

2.3.1 EMG Signal Description . . . . . . . . . . . . . . 12

2.3.2 Types of EMG Recordings . . . . . . . . . . . . . . . 13

3 Methods . . . . . . . . . . . . . . . . . . . 15 
3.1 Synthetic sEMG Signal . . . . . . . . . . . . . . . . . . 15

3.1.1 General Information on MUAP Waveform Models . . . . . . . 16

3.1 .2 MUAP Waveform Model . . . . . . . . . . . . . . 16

3.1 .3 sEMG Signal Model . . . . . . . . . . . . . . . . . . . 17

3.2 Experimental sEMG Signal Data Collection . . . . . . . . . 18

3.3 Fourier Series Method . . . . . . . . . . . . . . . . . . 18

3.4 General Perturbative Approximate Series Expansion . . . . . . . . . 19

3.5 Pseudocode for Perturbative Approximate Series Expansion _... . 21

3.6 Perturbative Approximate Series Expansion for Decomposition of sEMG Signal . . . . . . . . . . . . . . . . . . 22

3.7 Statistical Analysis . . . . . . . . . . . . . . . . . . . . 22

4 Results . . . . . . . . . . . . . . . . . . . . . . . . 24

4.1 Decomposition of Superimposed MUAPs . . . . . . . . . . 24

4.2 Decomposition of MUAPTs $\ldots \ldots \ldots \ldots \ldots \ldots$

4.3 Decomposition of Experimental Data . . . . . . . . . . . 34

5 Discussion . . . . . . . . . . . . . . . . . . . . . . . 4 4

5.1 Implications . . . . . . . . . . . . . . . 42

5.2 Limitations and Future Work _ . . . . . . . . . . . . . . 42

5.3 Conclusion . . . . . . . . . . . . . . . . . . . . 43

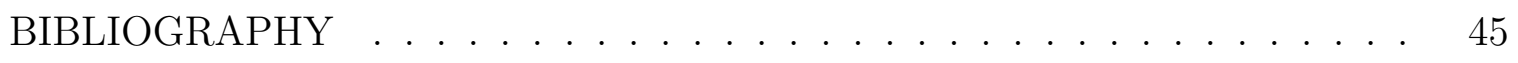
APPENDICES

A Synthetic MUAP Model . . . . . . . . . . . . . . . . 49

B Synchronous MUAP Decomposition . . . . . . . . . . . . 50

C Synthetic sEMG MUAP Decomposition . . . . . . . . . . 53

D Real sEMG MUAP Decomposition $\ldots \ldots \ldots \ldots \ldots$ 


\section{LIST OF TABLES}

Table

4.1 Parameters describing MUAPTs present in the synthetic sEMG signal. 28

4.2 Error table comparing actual and estimated firing rates for the MUAPTs present in the synthetic sEMG signal. . . . . . . . . . . . . . . 34 


\section{LIST OF FIGURES}

Figure

Page

1.1 Decomposition of EMG Signal (plot constructed using data from [1])

1.2 Steps for Decomposition of EMG Signal . . . . . . . . . . . . . 4

2.1 Anatomy of Skeletal Muscle $[3] \ldots \ldots \ldots$. . . . . . . . . 7

2.2 Motor unit is the motor neuron and the muscle fibers it innervates at neuromuscular junctions [3]. . . . . . . . . . . . . . . . . 9

2.3 Muscle fiber action potential $[3] \ldots \ldots$. . . . . . . . . 11

4.1 Component functions for four MUAPs synchronously firing with shape

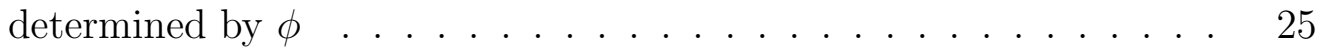

4.2 Compound function. $\psi(t) \ldots \ldots \ldots 26$

4.3 Contribution estimates from perturbation and Fourier series compared with actual distribution. . . . . . . . . . . . . 27

4.4 MUAP templates $f_{1}, f_{2}, f_{3}$ used to create compound EMG signal composed of MUAPTs $u_{1}, u_{2}, u_{3}$, respectively . . . . . . . . . . . . 28

4.5 Compound synthetic sEMG signal with the three MUAPTs composing it shown below. $u_{1}$ is blue, $u_{2}$ is red, and $u_{3}$ is green. . . . . . 29

4.6 Component functions. . . . . . . . . . . . . . . . . . . . . . 29

4.7 Estimated constituent functions, estimated constituent functions after thresholding $\beta$, and actual constituent functions for motor unit

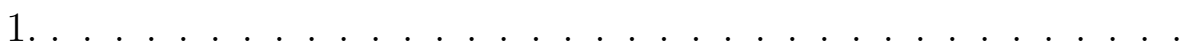

4.8 Estimated constituent functions, estimated constituent functions after thresholding $\beta$, and actual constituent functions for motor unit 2. . . . . . . . . . . . . . . . . .

4.9 Estimated constituent functions, estimated constituent functions after thresholding $\beta$, and actual constituent functions for motor unit 3. . . . . . . . . . . . . . . . . 
4.10 Frequency distribution of component functions after thresholding for synthetic sEMG signal. . . . . . . . . . . . . . . . . .

4.11 Comparison of the synthetic sEMG signal with the perturbation estimates reconstruction post-threshold. . . . . . . . . . 33

4.12 sEMG signal from biceps brachii. . . . . . . . . . . . . 34

4.13 Templates for MUAP shapes present in sEMG signal. . . . . . . . . 35

4.14 EMGLAB decomposition results shown for a single MUAP template shape present in the sEMG in Figure $4.12 \ldots \ldots \ldots$

4.15 Representative component functions for a single MUAP template shape with shifts of $2.5 \mathrm{~ms} \ldots \ldots \ldots \ldots$

4.16 Estimated constituent functions, estimated constituent functions after thresholding, and actual constituent functions for motor unit 2.

4.17 Estimated constituent functions, estimated constituent functions after thresholding, and actual constituent functions for motor unit $3 . \quad 38$

4.18 Estimated constituent functions, estimated constituent functions after thresholding, and actual constituent functions for motor unit 5 .

4.19 Estimated constituent functions, estimated constituent functions after thresholding, and actual constituent functions for motor unit 6 . 


\section{Introduction}

A fundamental part of being human is movement; precise control over our physical bodies allows for biking, dancing, playing catch, and other endless pursuits. It is how our bodies interact with the physical world around us. The deterioration of motor skills due to neuromuscular disorders drastically changes lives, such as difficulty feeding of oneself due to hand tremors. Muscular health can be better understood through study of the electrical activity of muscles, called electromyographic (EMG) signals. The EMG signal records action potentials from muscle fibers, which are organized into motor units (MUs) due to innervation by the same nerve. Many neuromuscular disorders affect the firing rates and amplitudes of motor unit action potentials (MUAPs), so being able to detect these changes would be useful. The purpose of this thesis is to present a technique for decomposing EMG signals into the firing times of constituent MUAPs to aid in the diagnosis of neuromuscular diseases.

\section{$1.1 \quad$ Motivation}

Electromyography is a common technique for evaluation of neuromuscular diseases including amyotrophic lateral sclerosis (ALS), myasthenia gravis, and Isaac's syndrome [4]. For neuromuscular disorders, early diagnosis is essential for treatment and understanding of disease progression. The pathophysiology of neuromuscular diseases may be myopathic, neurogenic, or affecting the neuromuscular junctions. Electromyography provides insight into the health of these systems by recording the electrical activity of skeletal muscles. In a clinical setting, electromyography recordings are commonly analyzed qualitatively by an expert using visual and auditory pattern recognition [4]. However in research there is movement toward quantitative analysis 


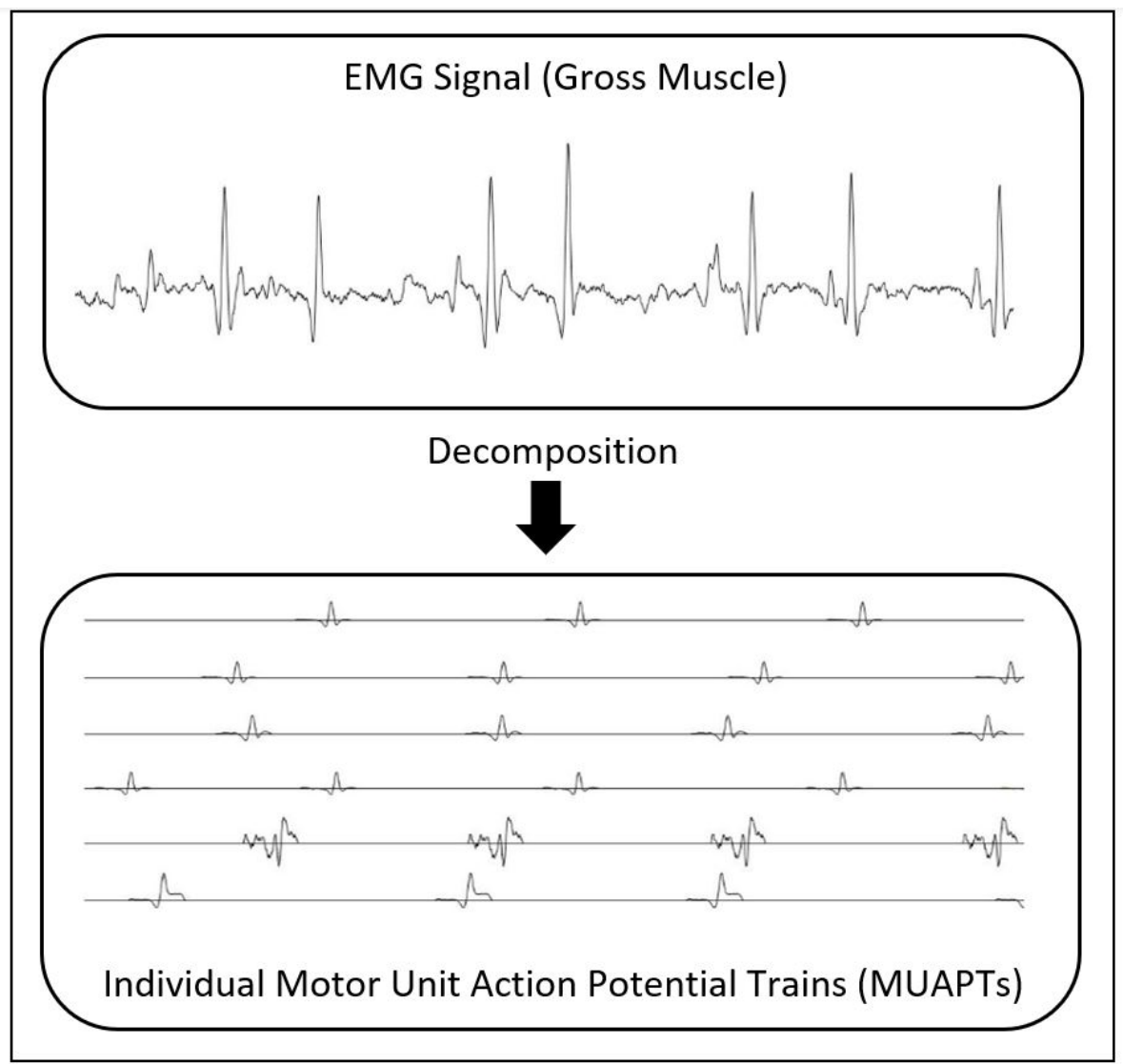

Figure 1.1: Decomposition of EMG Signal (plot constructed using data from [1])

and decomposition of EMG signals to provide details not apparent from the macro level. Properties of decomposed EMG signals such as number of MUAPs present, firing rate, and amplitude are critical for accurate diagnosis [5, 6]. Quantitative estimates of the number of MUAPs present provide useful information about motor unit recruitment [6]. Intramuscular EMG (iEMG) works relatively well but is invasive and has limitations, so achieving successful decomposition of surface EMG (sEMG) would be beneficial. sEMG decomposition allows for noninvasive characterization of motor units and study of the morphological organization of muscle fibers within intact muscle. This is useful for research on muscle activation as well as clinically for diagnosis and understanding disease progression. Another benefit of sEMG is that it can be used for investigation of motor control by nonclinical researchers [7]. 
The superposition of multiple signal constituents is one of many difficulties when decomposing EMG signals [5]. By trying out a decomposition technique that is more robust to non-orthogonal signals, decomposition of superimposed EMG signals may be better handled than with other algorithms. The motivation behind this thesis is to decompose EMG signals into the constituent motor unit action potentials to aid in diagnosis of neuromuscular disorders and diseases using a perturbation based decomposition.

\subsection{Previous Work}

The decomposition of sEMG signals has been investigated by numerous researchers since 1985 [8] and was likely inspired by the high success of iEMG decomposition [9]. Approaches for decomposition of sEMG into constitutive MUAPTs include nonlinear dynamic methods, Fourier transform, wavelet transform, other time-frequency approaches, and the Wigner-Ville Distribution [6]. Many decomposition methods $[10,11]$ use template-matching for recognition of MUAPs which requires prior identification of templates present in the sEMG signal and assumes that the MUAP shape is stationary [12]. Decomposition of sEMG is usually performed for low-level voluntary contraction signals, at less than $10 \%$ of maximal voluntary contraction. Low-level signals support the assumption that the MUAP shapes are stationary, as they are minimally affected by fatigue. The level of user interaction also varies between methods, with Kleine [10] using a highly user interactive multi-step technique, Garcia [13] using a semiautomatic decomposition technique to extract average firing rates, and Gazzoni [14] using a technique that automatically decomposes two to three real MUAPTs that are not superimposed. Between the various decomposition techniques, there are common stages of analytical procedures. After preprocessing the EMG signal to remove noise, the template or candidate MUAP waveforms are 
identified. Then, the EMG signal is decomposed into template MUAP waveforms and classified [5].

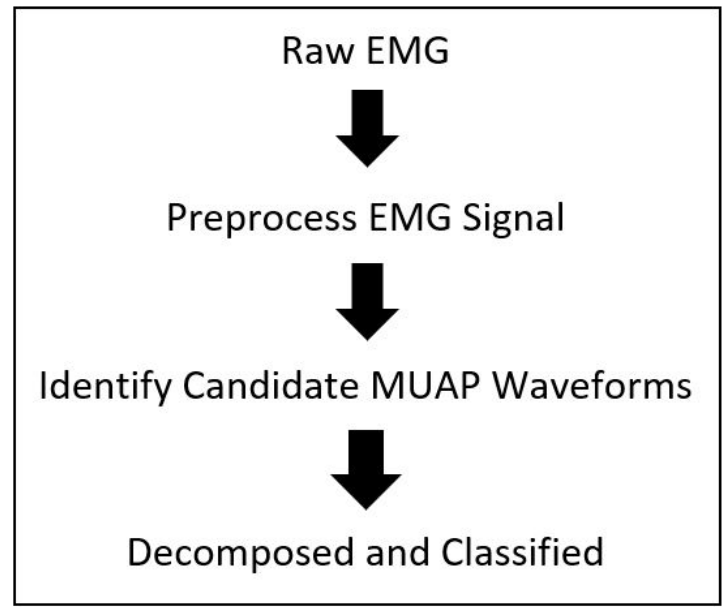

\section{Figure 1.2: Steps for Decomposition of EMG Signal}

\subsection{EMG Decomposition Challenges}

There are challenges and limitations present within each stage of EMG decomposition. In the preprocessing stage, the main challenge is removal of noise due to external interference, motion artifact, poor signal-to-noise ratio and baseline drift. Determining candidate MUAPs is also difficult due to variation in MUAP waveforms within a motor unit, similarities in MUAP waveforms from different motor units, temporal overlap of MUAPs, and/or dynamic ranges of MUAP amplitudes [15]. MUAP templates have more similarity of shape for sEMG than for iEMG due to the low pass effects of the subcutaneous tissue and skin, which increases difficulty of decomposition. For sEMG, the shape changes have less impact as they average out between the many muscle fibers composing the motor unit. The complexity of the signal with regard to number of MUAPTs and duration of MUAPs affects temporal overlap and consequently the success of the decomposition. The large range of MUAP amplitudes due to distance from the electrode and anatomical considerations affect 
decomposition; minimum amplitude thresholds are often utilized. Decomposition of sEMG works best at low force levels with limited numbers of motor units [6]. The superposition of MUAPs is the major limitation for decomposition of sEMG signals accomplished by template-matching [7].

\subsection{Outline}

The organization of the paper is as follows: first, relevant background on the neuromuscular system and EMG recording techniques will be presented, then methods for application of perturbation based decomposition to the synthetic and experimental signals, and finally results including comparison with another decomposition method for validation. 


\section{Background}

\subsection{The Human Neuromuscular System}

The neuromuscular system consists of the nervous system, muscular system, and neuromuscular junctions that connect the two.

\subsubsection{The Nervous System}

The nervous system includes the central nervous system (CNS) which is the brain and spinal cord, and the peripheral nervous system (PNS) which is the communication system between the CNS and body. Within the PNS is sensory division for sending sensory information from the body to the CNS, and the motor division for sending movement information from the CNS to the body. The motor division interfaces with the muscular system, controlling both somatic (voluntary) and autonomic (involuntary) movement through impulses delivered by motor neurons to muscle fibers. $[3]$.

\subsubsection{The Muscular System}

The human muscular system is composed of three types of muscular tissue: skeletal, cardiac, and smooth. Muscle tissue is a specialized contractile tissue, and its primary function is contraction to produce force for movement of the body. Other functions include posture stability, heat generation, circulation, and aiding digestion. Skeletal muscle attaches to bones for movement of limbs and other structures and is composed of skeletal muscle fibers, nerve fibers, blood vessels, and other connective tissue. 
Contraction of skeletal muscle is controlled by the somatic division of the PNS, while both cardiac muscle and smooth muscle are under autonomic control.

\subsubsection{Gross Skeletal Muscle Organization}

Skeletal muscle is organized into groupings of muscular tissue surrounded by connective tissue. At the macro level, the connective layer surrounding the entire muscle is the epimysium. Within the epimysium are groupings of (up to 150) muscle fibers called fascicles, each surrounded by a perimysium [3]. Each individual muscle fiber is surrounded by a sarcolemma, and composed of myofibrils. Blood vessels are found between fascicles, while blood capillaries are found within each sarcolemma. The motoneurons attach to the surface of individual muscle fibers. The image below details the anatomy of this highly organized organ.

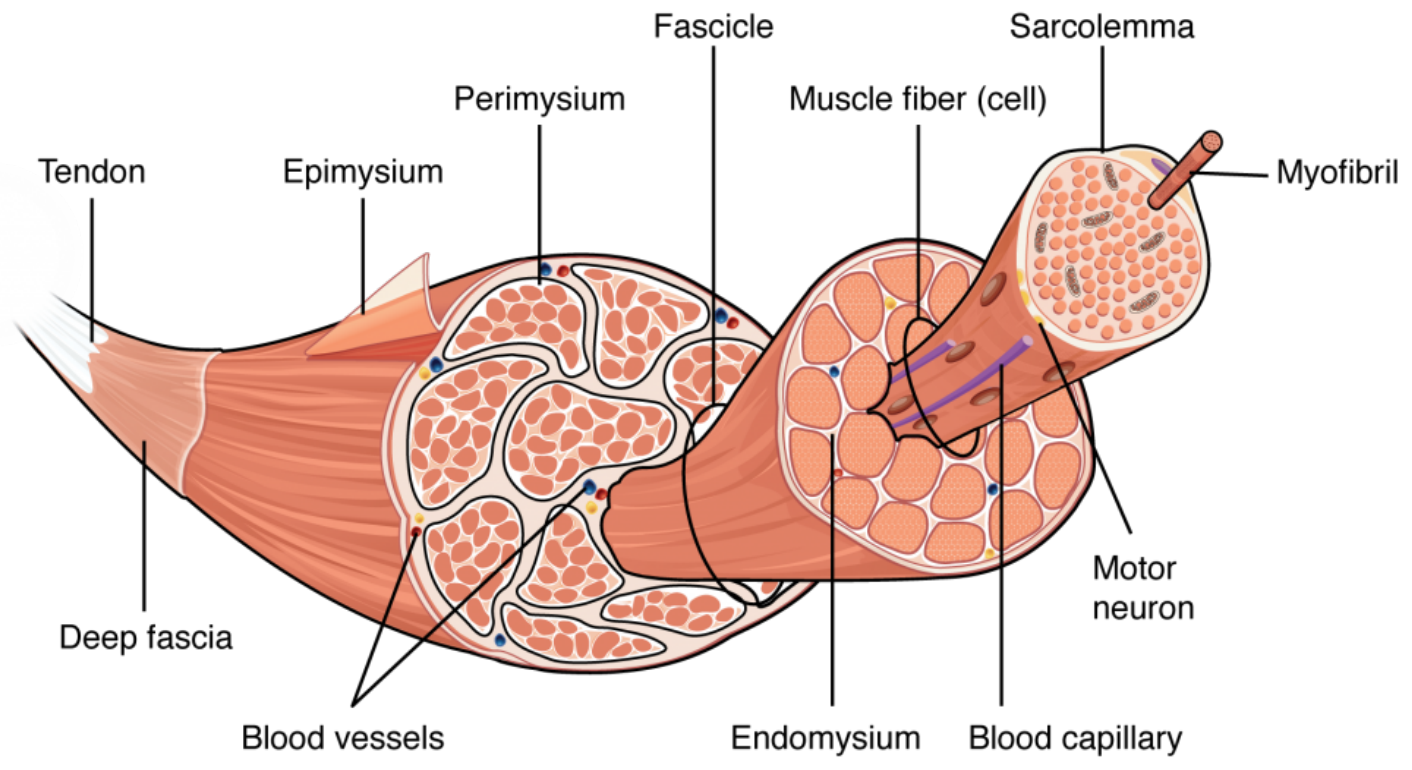

Figure 2.1: Anatomy of Skeletal Muscle [3] 


\subsubsection{Skeletal Muscle Fibers}

Skeletal muscle fibers are the cell type composing skeletal muscle. Within a single muscle fiber there are hundreds to thousands of myofibrils that contain the contractile elements of skeletal muscle called sarcomeres. Sarcomeres contain myofilaments which are the basis of contraction and pull together to create crosslinks. Each skeletal muscle fiber is excited by a single motor neuron, causing it to release calcium and begin the contraction process [3].

\subsubsection{Motor Units}

Each somatic motor neuron innervates a group muscle fibers, ranging from few to hundreds [16] and activates them all simultaneously in an 'all or none' manner. The number of muscle fibers per motoneuron is correlated with precision of motor control; muscles with fine motor control have fewer muscle fibers innervated per motoneuron. The grouping of an individual motor neuron with the muscle fibers it innervates are a motor unit [4]. When a motor neuron fires, all muscle fibers within that motor unit will contract synchronously.

\subsubsection{The Neuromuscular Junction}

The neuromuscular junction is the location of communication between a motor neuron and a muscle fiber. It is the chemical synapse between the axon terminal of the motor neuron and the sarcolemma of the muscle fiber, where the signal to initiate muscle fiber contraction is transmitted. Communication between neuron and muscle fibers occurs via neurotransmitters that diffuse across the gap [3]. 


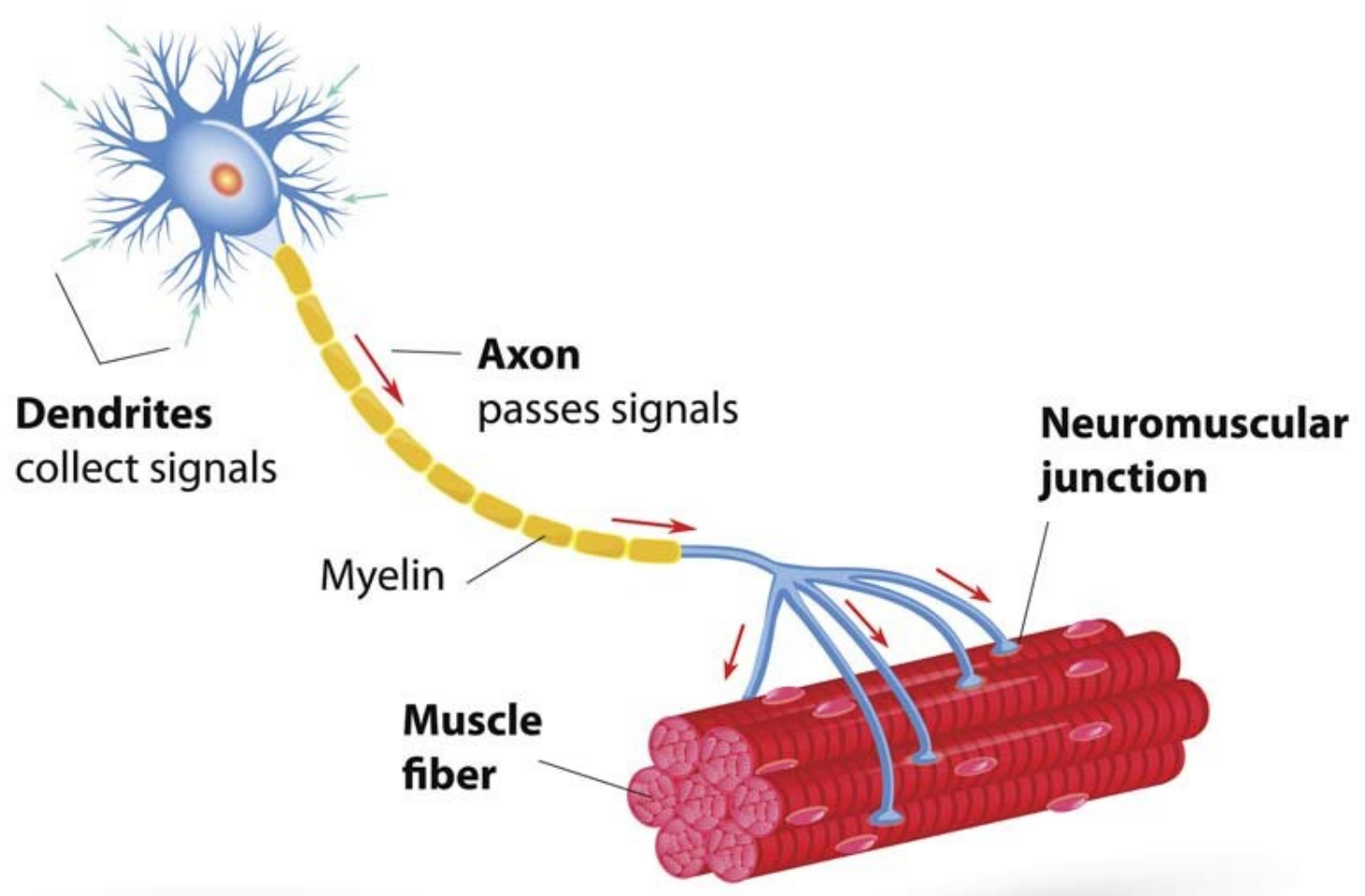

Figure 2.2: Motor unit is the motor neuron and the muscle fibers it innervates at neuromuscular junctions [3].

\subsection{Skeletal Muscle Activation}

The movement of charged ions and neurotransmitters within the neuromuscular system form a complex messaging system that initiates of contraction. The sEMG signal is measured from the net electrical activity associated with ion flow, so it is important to study the physiological pathways to understand the origin of the signal.

\subsubsection{Physiology of Muscle Fiber Activation}

Skeletal muscle contraction initiates as an impulse in the CNS, which travels down a somatic motor neuron in the PNS as an action potential until it reaches the neuromuscular junction. At the neuromuscular junction, the impulse crosses the synapse chemically as the neurotransmitter Ach. Ach activates the muscle fiber by binding 
with the $\mathrm{Na}^{+}$receptors on the sarcolemma, opening sodium channels and causing depolarization via an influx of $\mathrm{Na}^{+}$into the muscle fiber. The depolarization spreads down the length of the muscle fiber as a wave, causing the release of $\mathrm{Ca}^{2+}$ from the sarcoplasmic reticulum and initiating excitation contraction coupling. Due to a delayed response to the depolarizing wave, $K^{+}$channels on the sarcolemma open and $K^{+}$rushes out to repolarize the cell and return it to the resting potential. The propagation of depolarization followed by repolarization down the length of the muscle fiber is known as a muscle fiber action potential.

\subsubsection{Muscle Fiber Action Potential (MFAP)}

The muscle fiber action potential is due to the changes in membrane voltage as the charged ions sodium and potassium cross the membrane. After the sodium channels are opened, sodium rushes into the cell due to a concentration gradient and the membrane potential consequently increases in positivity. This depolarization (due to sodium entering the muscle fiber) causes the upstroke of the muscle fiber action potential, while potassium rushing out of the cell for repolarization causes the downstroke.

\subsubsection{Motor Unit Action Potential (MUAP)}

A MUAP is the summation of the MFAPs within a motor unit. Because muscle fibers within a motor unit are all activated at once by the same neuron, the MFAPs are temporally overlapped and are detected as a single unit known as a MUAP. Anatomically, a MUAP is the combined action potential signal from all the muscle fibers in the motor unit. In an EMG signal, the MUAP is defined slightly differently as the action potential summation from the muscle fibers in the motor unit within 


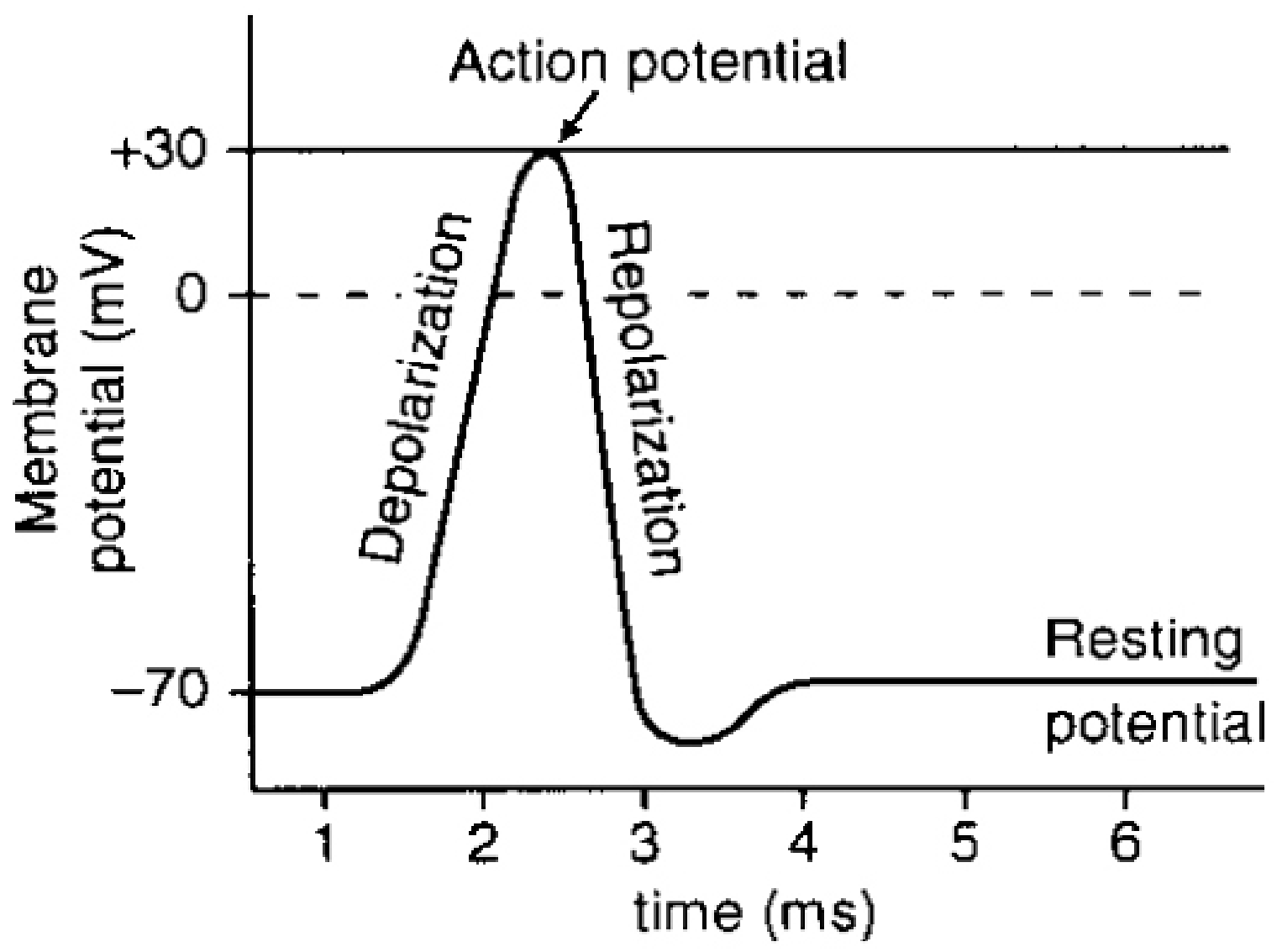

Figure 2.3: Muscle fiber action potential [3]

recording proximity of the electrode (this paper will use this definition) $[17,5]$. This difference is due to electrode spatial recording limitations.

The MUAP characteristics are affected by the size, conduction velocity, and positioning of the detection electrode relative to the muscle fiber. The magnitude of the MUAP is affected by the fiber diameters of its constituent muscle fibers, with larger fiber diameters creating larger MFAPs and smaller fiber diameters creating smaller MFAPs [18]. The magnitude is also affected by the electrode distance from each muscle fiber, with muscle fibers positioned closer to the electrode recording a higher value. The duration of the MFAP is affected by the conduction velocity of the muscle fiber, which is the speed that the AP travels down the muscle fiber [18] which in turn affects the MUAP. The shape of the MFAP waveform also varies with the distance 
between the electrode recording side and the innervation zone [19].

\subsubsection{Motor Unit Action Potential Train (MUAPT)}

For sustained muscle contraction, motor units repeatedly fire action potentials to create a train of motor unit action potentials, or a MUAPT [19]. To increase force of contraction, the muscle must either activate muscle fibers more frequently or recruit more units [18]. The frequency of MFAP activation is the firing rate (which is limited by the absolute refractory period). Firing rate of normal muscle is $4-12 \mathrm{~Hz}$ until another motor unit is recruited. Recruitment typically follows a 5:1 pattern, which is the ratio of frequency to number of MUAPs firing (at $10 \mathrm{~Hz}$ a second MUAP recruited, at $15 \mathrm{~Hz}$ a third MUAP recruited) [7]. The firing rate of MUAPT ranges from $6 \mathrm{~Hz}$ to $40 \mathrm{~Hz}[15,16]$.

Numerous neuromuscular diseases affect the firing rates and interval variability of motor units. For myopathic disorders, firing frequency of motor units has been shown to be higher than control groups while variability in interval length is not significantly

different. It is believed the increased firing rates are compensation for low muscle strength. For neuropathic disorders, there are significant increases in both firing rates and MUAP interval variability. The cause for variability in the interval between MUAPs is hypothesized to be due to irregularity in the threshold potential value [20].

\subsection{Electromyography Signal and Recording Techniques}

\subsubsection{EMG Signal Description}

Electromyography records the electrical activity produced by skeletal muscle as an indication of neuromuscular activation [21]. At rest, each skeletal muscle fiber mem- 
brane potential is negative due to differences in intracellular and extracellular ion concentration and composition [19]. When the skeletal muscle fiber membrane potential is depolarized to the activation threshold, a MFAP propagates down the length of the muscle fiber as a depolarizing wave. For each muscle fiber, the propagating action potential generates an electromagnetic field. The electric field in the muscle and the electric potential on the surface of the skin exist whether or not they are recorded [16]. The electrode will measure the net electric potential due to contributions from

all MUAPTs in the spatial recording vicinity. [18]. Bipolar electrodes are commonly used with a differential amplifier to suppress noise; if monopolar electrodes are used, the signal will have a different shape.

For EMG recording, there are two types of activation of the muscle: voluntary and involuntary. For voluntary EMG, the muscle is activated natively by the neuron, and for involuntary or evoked EMG, external electrical stimulation is applied to initiate action potentials in the muscle.

\subsubsection{Types of EMG Recordings}

There are two main types of EMG recordings: surface EMG and intramuscular EMG. For sEMG recordings, electrodes placed on the surface of the skin measure the induced electric field as voltage [21]. The sEMG signal is the summation of the MUAPTs in the vicinity of the electrode, as each motor unit in the vicinity of the electrode fires intermittently to form MUAPTs [5].

For intramuscular/needle EMG recording, a needle electrode is inserted into the muscle to record the action potential of a single muscle fiber. Small movements and damage to the muscle due to insertion of indwelling electrodes influence the shapes of action potentials [7]. Compared with needle EMG, the application of sEMG elec- 
trodes is simpler, the subject feels less discomfort, and medical supervision is not required. There is higher repeatability for sEMG measurements as the variations in individual MUAP waveforms are averaged [6]. However, for this same reason MUAP shapes of different motor units may be overly similar and have inadequate temporal differences [6]. 


\section{Methods}

The perturbation based decomposition method was applied to sEMG signals to determine firing times and amplitudes of the MUAP waveform constituents. These characteristics are useful for understanding neuromuscular disease progression and diagnosis. In sEMG signals, MUAP waveforms are often superimposed which increases difficulty of decomposition. The application of the perturbation based decomposition to sEMG signals will be discussed and evaluated in this paper.

Prior to applying the perturbation decomposition technique, the MUAP waveform shapes present in the sEMG signal must be known. The MUAP templates used for construction of the synthetic sEMG signal are based on Hermite-Rodriguez functions from literature. In the experimental section of this paper, the MUAP templates are derived from the sEMG signal by the EMGLAB algorithm [22].

\subsection{Synthetic sEMG Signal}

As proof of concept for the application of the perturbation based decomposition to real sEMG signals, it was tested on synthetic sEMG signals. The compound synthetic sEMG signal was created as the summation of template MUAP waveforms firing at

fixed rates over the duration of the signal. Then, the decomposition technique was applied using the template MUAP waveforms shifted over the length of the sEMG as the component functions. 


\subsubsection{General Information on MUAP Waveform Models}

The shape and amplitude of each MUAP waveform will vary due to fiber geometry, conduction velocity, and electrode location. Each MUAP waveform shape can be assumed to remain constant if the electrode positioning is constant, the firing rate is constant, and there are no significant biochemical changes in the muscle tissue such as fatigue $[19,6]$. Because the decomposition returns an amplitude for each MUAP template, it is unnecessary to incorporate amplitude into the component functions used during decomposition.

In sEMG signal recordings, differences between motor units become less pronounced due to low pass filtering effects of the skin and subcutaneous tissues and a farther recording distance (as compared with iEMG). It is theoretically possible to use a small set of MUAP template waveforms to represent a larger number of motor units, allowing for representation of multiple motor units with the same template [6]. This would require the perturbation based decomposition results to be further decomposed into MUAPTs by analyzing varying amplitudes and firing rates. For simplicity in this paper, the main template shapes will each only represent only one motor unit to avoid further decomposition of the results.

\subsubsection{MUAP Waveform Model}

Hermite-Rodriguez functions are commonly used for representation of differentially derived MUAP waveforms to promote simplicity of sEMG simulations. In Homomor-

phic Deconvolution [15], a model for the MUAP based on the Hermite Rodriquez series expansion was developed, with the basis functions defined as 


$$
\omega_{n}(\alpha, t)=\frac{1}{\sqrt{2^{n} n !}} H_{n}(t / \alpha) \frac{1}{\sqrt{\pi} \alpha} e^{(-t / \alpha)^{2}}
$$

where $H_{n}$ is the Hermite polynomial of order n. After the time scaling is removed, the basis functions become

$$
v_{n}(\zeta)=\omega(1, \zeta)=\frac{1}{\sqrt{\pi 2^{n} n !}} H_{n}(\zeta) e^{-\zeta^{2}}
$$

and each MUAP is modelled as a series of the above basis functions

$$
f(\zeta)=\sum_{n=1}^{N} a_{n} v_{n}(\zeta)
$$

In this paper, normalized basis functions up to the second order were linearly combined and the coefficients $a_{1}$ and $a_{2}$ represented in terms of $\phi$.

$$
\left.f(\zeta)=\sin (\phi) \frac{v_{1}(\zeta)}{\left\|v_{1}\right\|}\right]+\cos (\phi) \frac{v_{2}(\zeta)}{\left\|v_{2}\right\|}
$$

where $\phi$ is the angle between vectors $v_{1}$ and $v_{2}$. For $\phi=\pi / 2$, the first order vector is returned $f=v_{1}$ with a biphasic shape, while for $\phi=0$, the second order vector is returned $f=v_{2}$ with a triphasic shape. While the first order vector and second order vector are orthogonal with $v_{1} \cdot v_{2}=0$, intermediate values of $\phi$ will create nonorthogonal MUAP shapes [15].

\subsection{3 sEMG Signal Model}

The sEMG signal may be modelled as the summation of MUAPTs which are in turn the summation of delayed versions of a MUAP. For the $j$ th MUAPT composed of 
MUAP $f(\zeta)$ with amplitude $\kappa_{j}$ and firing times $t_{i j}$

$$
M U A P T_{j}=u_{j}(t)=\sum_{i} \kappa_{j} f\left(t-t_{i j}\right)
$$

where $t_{i j}$ is the time-shift for the $i$ th MUAP in the $j$ th MUAPT Thus, the sEMG signal $\psi(t)$ may be represented as

$$
\psi(t)=\sum \sum_{j} M U A P T_{j}(t)=\sum \sum_{i} \kappa_{j} f\left(t-t_{i j}\right)
$$

\subsection{Experimental sEMG Signal Data Collection}

In a study by J-Y Hogrel, EMG signals were recorded from the biceps brachii during sustained low level contractions using laplacian surface electrodes. The contractions aimed to be isometric and isotonic. In the study, the constituent MUAPs parameters were analyzed for stability over time to investigate the assumption they are constant for non-fatiguing contractions. The sampling rate was $10 \mathrm{kHz}$ and bandpass filtering was applied from $10 \mathrm{~Hz}$ to $1 \mathrm{kHz}$. An EMGLAB algorithm [22] was applied to the signal to determine the constituent waveforms, then considerable manual editing was performed for decomposition. The filtered sEMG signal, constituent MUAP wave-

forms, and firing times considered to be the "gold standard" of sEMG decomposition were provided [1].

\subsection{Fourier Series Method}

The Fourier series is a technique for decomposition of a compound signal into constituent signals, but has a major limitation of the requirement of orthogonality for the 
constituent signals. Due to MUAPs often being nonorthogonal and temporally overlapped within the sEMG signal, the Fourier series cannot provide a precise estimation of the templates present due to nonorthogonality.

\subsection{General Perturbative Approximate Series Expansion}

The perturbative approximate series expansion, also termed perturbation based decomposition, was developed by Szlavik as a technique to estimate the distribution of nerve fiber diameter sizes contributing to a compound-evoked potential [23]. The technique has since been applied to the characterization of neurotransmitter receptor activation [24]. Additional inverse problems in electrophysiology are currently being pursued, including the topic of this paper [25].

For the compound function $\psi(t)$, the contributions $\tilde{\beta}_{n}$ from component functions $\lambda(t)_{n=1}^{m}$ may be approximated using the perturbative approximate series expansion. The component functions are not required to satisfy the orthogonality condition or form a basis for the function space. The contribution $\tilde{\beta}_{n}$ from each component function $\lambda_{n}$ is assumed to be perturbed by the contributions of the other component functions in the set.

$$
\psi(t)=\sum_{n=1}^{m} \tilde{\beta}_{n} \lambda_{n}(t)
$$

In the frequency domain, the compound signal $\Psi(f)=F[\psi(t)]$ is composed of frequency components from each of the component functions $\Lambda_{n}(f)=F\left[\lambda_{n}(t)\right]$ perturbed by one another by a estimable degree $\epsilon_{i, j}$. This can be written as 


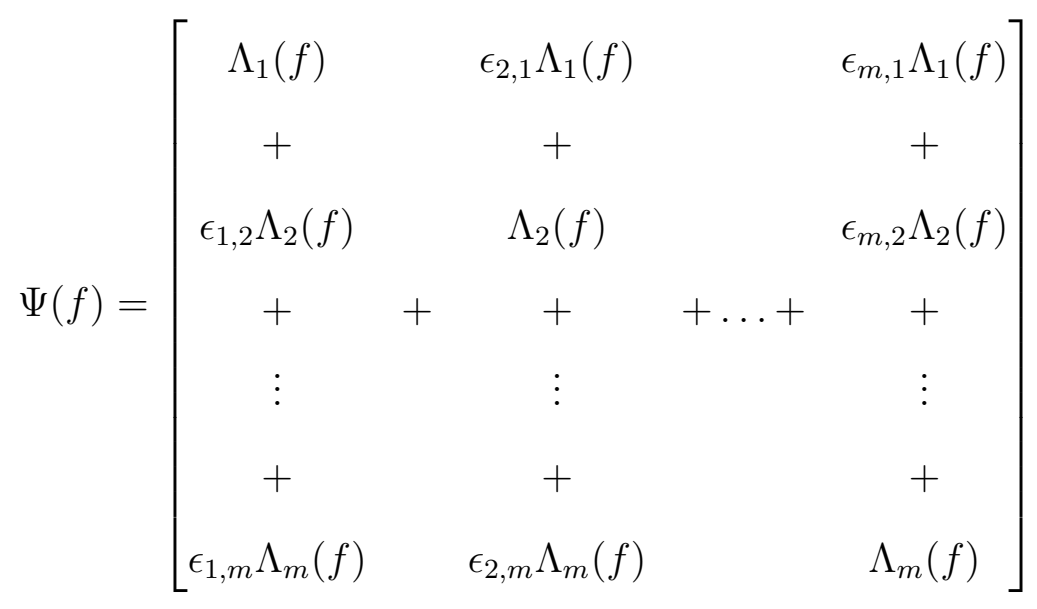

where $\epsilon_{i j}$ represents the perturbation of the $i$ th component function by the $j$ th component function. Equation 3.8 can be rewritten as

$$
\begin{gathered}
\Psi(f)=\sum_{n=1}^{m} \widetilde{B_{n}} \Lambda_{n}(f) \\
\widetilde{B_{n}}=\epsilon_{i, n}+\ldots+\epsilon_{n-1, n}+1+\ldots+\epsilon_{m, n}
\end{gathered}
$$

Rearranging the above expression and evaluating at specific frequencies $f_{k}$ for $k=$ $1 . . . l$ yields

$$
\Psi\left(f_{k}\right)-\sum_{i=1}^{m} \Lambda_{i}\left(f_{k}\right)=\sum_{i=1}^{m} \sum_{j=1}^{m} \epsilon_{i, j} \Lambda_{j}\left(f_{k}\right)
$$

The linear system of equations may be solved in the least squares sense for $\epsilon_{i, j}$ as long as the number of specific frequencies $l$ creates an overdetermined system with $l>m^{2}-m$. Using Equation 3.10 with $\epsilon_{i, j}$, the estimator in the frequency domain may be solved for. Then, the real-valued estimator $\tilde{\beta}_{n}$ which scales each constituent function as per Equation 3.7 can be found using Equation 3.12 below. 


$$
\tilde{\beta}_{n}=\left|\widetilde{B_{n}}\right|=\sqrt{\left(\widetilde{B_{n}}\right) \cdot\left({\widetilde{B_{n}}}^{*}\right)}
$$

\subsection{Pseudocode for Perturbative Approximate Series Expansion}

Szlavik provided a MATLAB function for estimation of the distribution of component functions present in the compound function using the perturbation approximate series expansion. The compound function and component functions are used as inputs to the function, and the estimation of the contribution from each constituent function is computed.

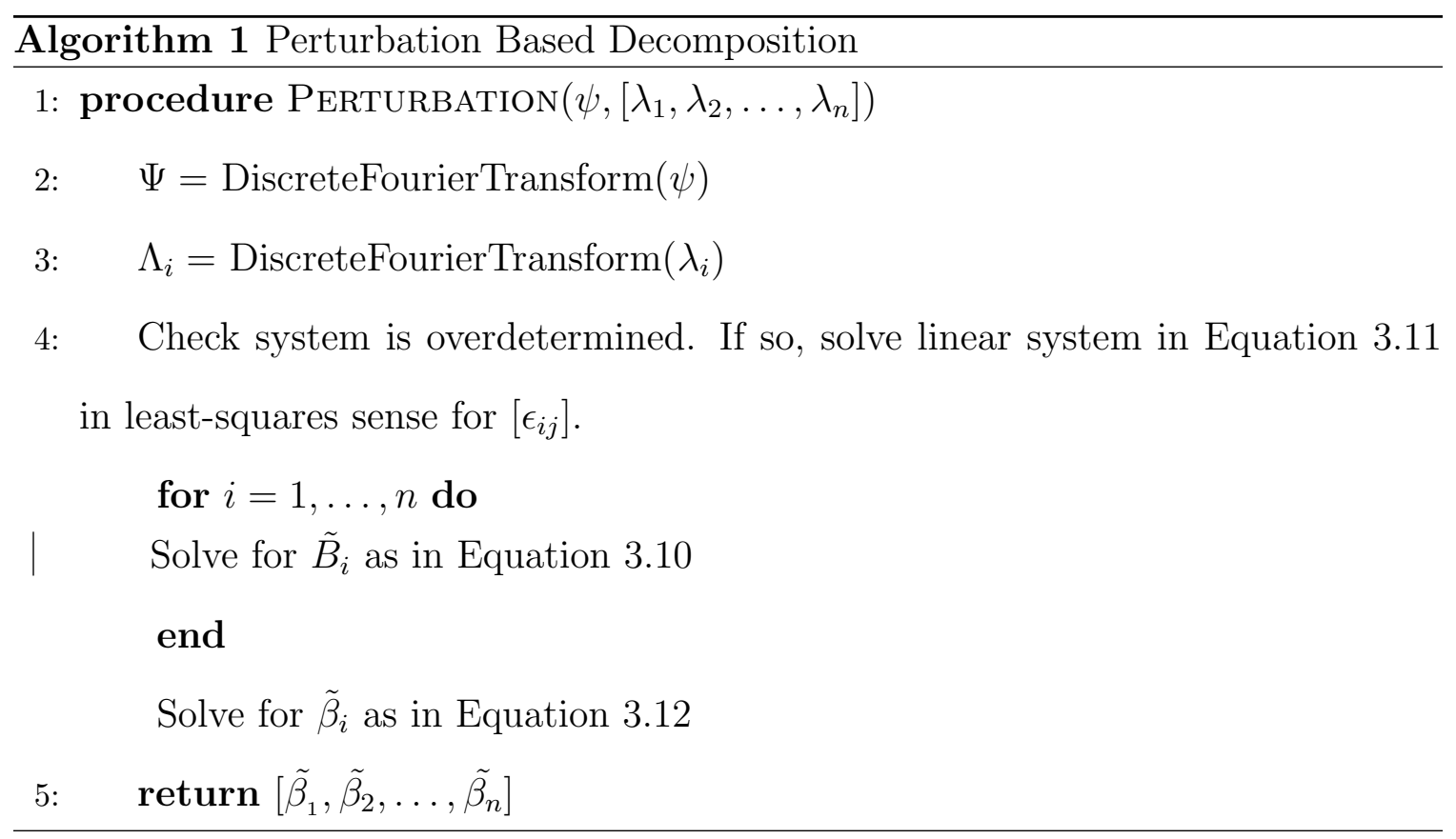




\subsection{Perturbative Approximate Series Expansion for Decomposition of sEMG Signal}

The perturbative decomposition method developed by Szlavik can be applied to sEMG signals, either synthetic or experimental, to return the firing times and amplitudes of the constituent MUAPs. For a sEMG signal composed of $p$ motor units, the compound potential $\psi(t)$ can be reconstructed as the summation of MUAPTs $u_{j}$ for $j=1 \ldots p$

$$
\begin{gathered}
\psi(t) \approx u_{1}(t)+u_{2}(t)+\ldots+u_{p}(t) \\
u_{j}(t) \approx \tilde{\beta_{1, j}} f_{j}\left(t-t_{1, j}\right)+\tilde{\beta_{2, j}} f_{j}\left(t-t_{2, j}\right)+\ldots+\tilde{\beta_{i, j}} f_{j}\left(t-t_{i, j}\right)
\end{gathered}
$$

where $f_{j}(t)$ is the MUAP waveform shape associated with the $j$ th motor unit, $t_{i j}$ is the time-shift for the $i$ th MUAP in the $j$ th MUAPT, and $\beta_{i j}$ is the estimated contribution from the $i$ th MUAP in the $j$ th MUAPT.

Assumptions necessary for decomposition and reconstruction:

- MUAP shape within a MUAPT remains relatively constant

- Component functions are the only MUAP shapes present

- Linear summation of MUAPTs

\section{7 $\quad$ Statistical Analysis}

Assessment of accuracy is non-trivial due to errors such as imprecision in firing time identification, inability to distinguish MUAP waveforms, and superposition between 
waveforms. For synthetic data, the correct decomposition is precisely and accurately known, but this is not the case for experimental data. In the experimental sEMG data provided by EMGLAB, the annotated decomposition displays approximates firing times and MUAP waveform shapes but cannot be considered $100 \%$ accurate. Quantification of decomposition error is challenging due to imprecision in the firing times and amplitudes of MUAP estimates. 


\section{Results}

The ability of the algorithm to identify the amplitude and firing times of constituent MUAP waveforms was investigated. First, the amplitudes for four superimposed nonorthogonal MUAP waveform models were decomposed from a compound signal. Then a compound function composed of MUAPTs was decomposed to determine firing times and amplitudes for each MUAP waveform. Component functions that

fired over the length were used as inputs to the decomposition algorithm for each MUAP waveform type. The sEMG was decomposed into MUAPs and these results compared with the Fourier series decomposition. The decomposition was then applied to sEMG experimental data for verification.

\subsection{Decomposition of Superimposed MUAPs}

A compound sEMG potential was arbitrarily created as the superposition of four nonorthogonal MUAP waveform templates. The MUAP waveform model from equation 3.4 was used with the value of $\phi$ determining the biphasic, triphasic, or intermediate shape.

$$
\psi(t)=2 f_{\phi=\pi / 2}(t)+7 f_{\phi=\pi / 4}(t)+4 f_{\phi=\pi / 8}(t)+10 f_{\phi=\pi / 16}(t)
$$

Each component function represents the MUAP shape for a different motor unit, with all motor units firing synchronously. It is important for the decomposition to identify the amplitude of each MUAP accurately, as the amplitude has the potential to vary throughout a MUAPT due to biochemical changes in the muscle. While it is unlikely for all four MUAPs to fire synchronously, this simulation shows the effectiveness of 
the decomposition.

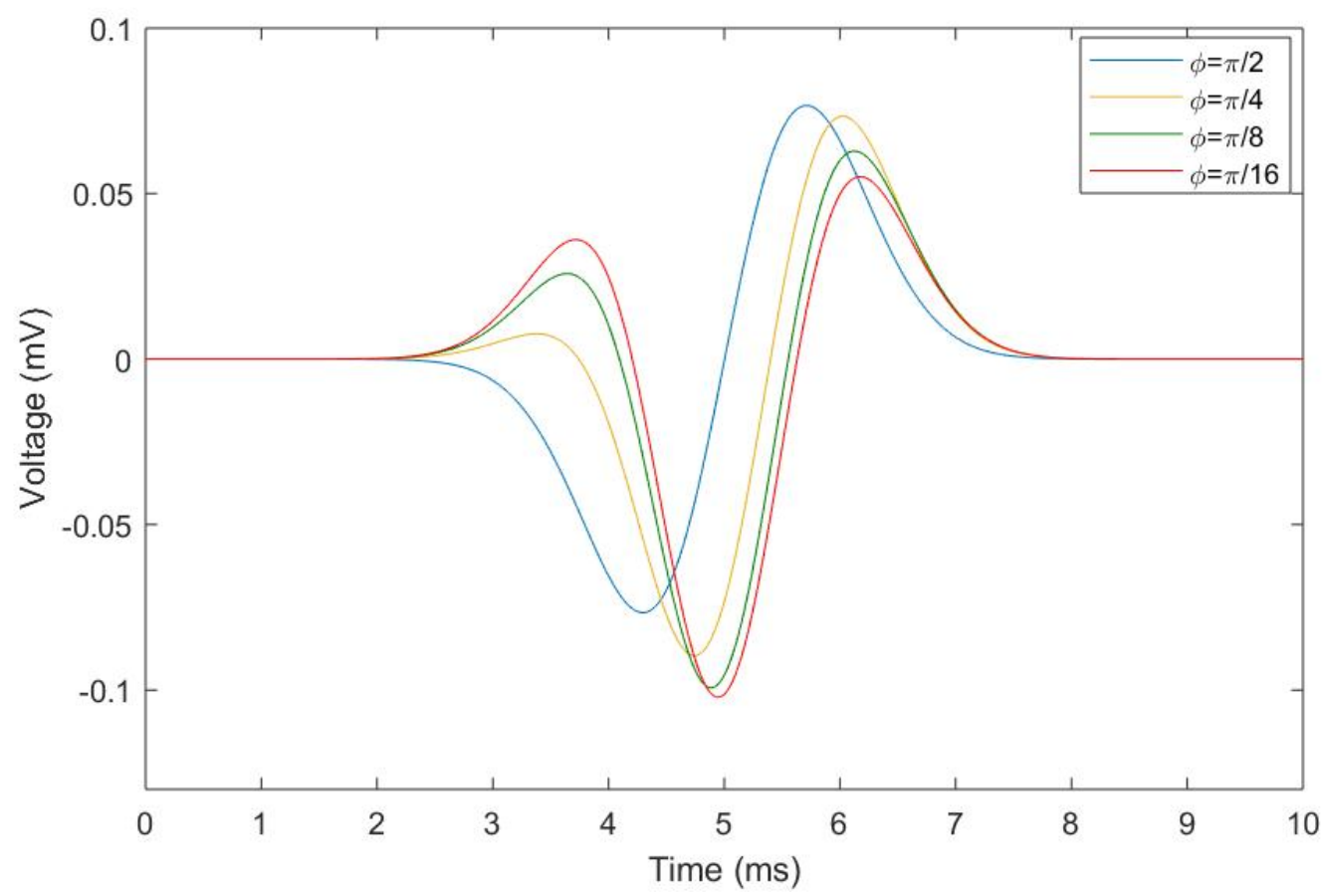

Figure 4.1: Component functions for four MUAPs synchronously firing with shape determined by $\phi$

The compound signal which is the sum of the four constituent functions as seen in equation 4.1 is below. 


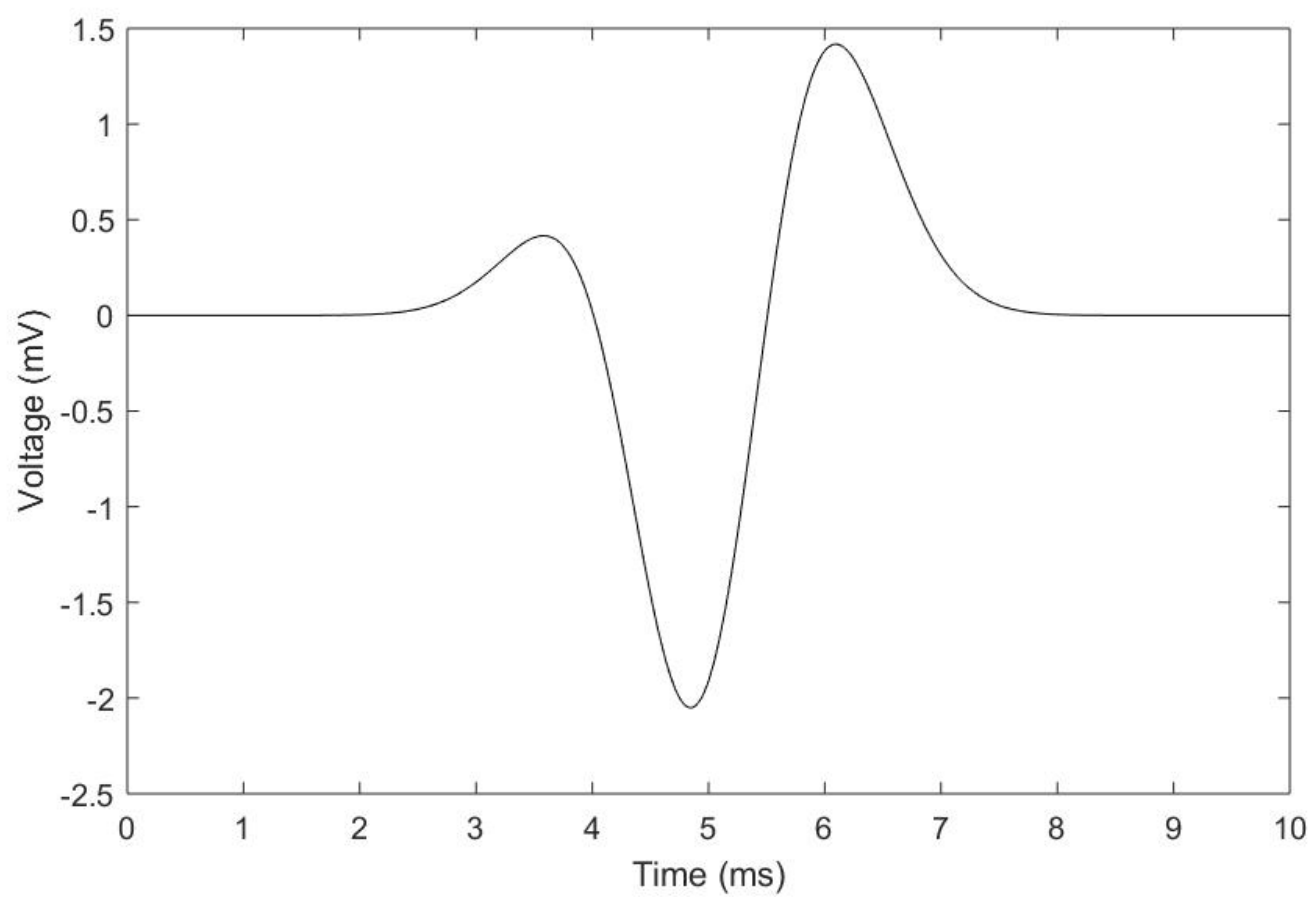

Figure 4.2: Compound function. $\psi(t)$

The estimates of the contribution from each component function were calculated using the Fourier series method and the perturbative approximate series expansion method. The plot comparing the estimates with the actual distribution is in Figure 4.3 below. 


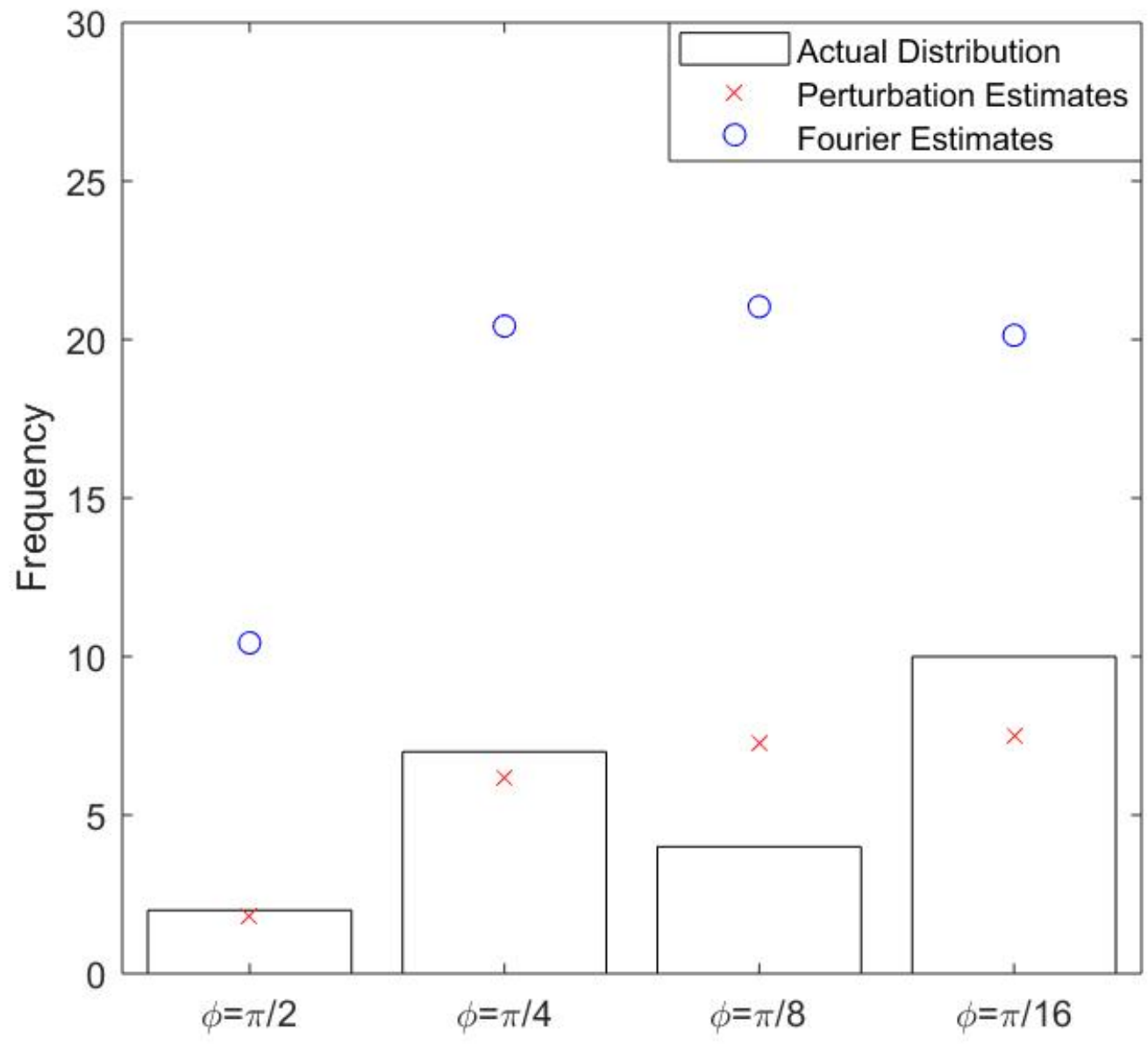

Figure 4.3: Contribution estimates from perturbation and Fourier series compared with actual distribution.

\subsection{Decomposition of MUAPTs}

A synthetic EMG compound signal was arbitrarily created that was composed of three MUAPTs incorporating dynamic range and fixed firing rates. The MUAPTs were formed from the MUAP templates (Figure 4.4) and modeled using equation 3.5.

The compound signal can be written as the summation of these MUAPTs as

$$
\psi(t)=u_{1}(t)+u_{2}(t)+u_{3}(t)
$$



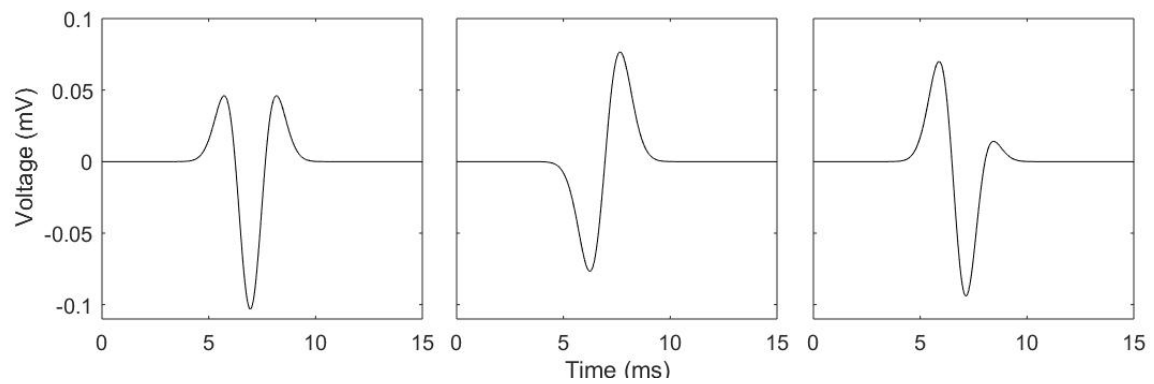

Figure 4.4: MUAP templates $f_{1}, f_{2}, f_{3}$ used to create compound EMG signal composed of MUAPTs $u_{1}, u_{2}, u_{3}$, respectively

Table 4.1: Parameters describing MUAPTs present in the synthetic sEMG signal.

\begin{tabular}{|cccc|}
\hline MUAPT & $\phi$ & Firing Time Spacing (ms) & Scale, $\kappa$ \\
\hline \hline$u_{1}$ & 0 & 20 & 3 \\
\hline$u_{2}$ & $\pi / 2$ & 24 & 6 \\
\hline$u_{3}$ & $-\pi / 5$ & 28 & 4 \\
\hline
\end{tabular}

where the MUAPTs can be represented in terms of shifted component MUAPs as

$$
\begin{aligned}
& u_{1}(t)=\kappa_{1}\left[f_{1}\left(t-t_{5}\right)+f_{1}\left(t-t_{10}\right)+\ldots+f_{1}\left(t-t_{45}\right)\right] \\
& u_{2}(t)=\kappa_{2}\left[f_{2}\left(t-t_{6}\right)+f_{2}\left(t-t_{12}\right)+\ldots+f_{2}\left(t-t_{48}\right)\right] \\
& \left.u_{3}(t)=\kappa_{3}\left[f_{3}\left(t-t_{7}\right)+f_{3}\left(t-t_{14}\right)\right]+\ldots+f_{3}\left(t-t_{49}\right)\right]
\end{aligned}
$$

where $t_{n}=(n-1) * 4 m s$ due to component functions spaced at $250 \mathrm{~Hz}$.

The compound signal is plotted in Figure 4.5 below, with the three constituent MUAPTs plotted as blue, red, and green. 


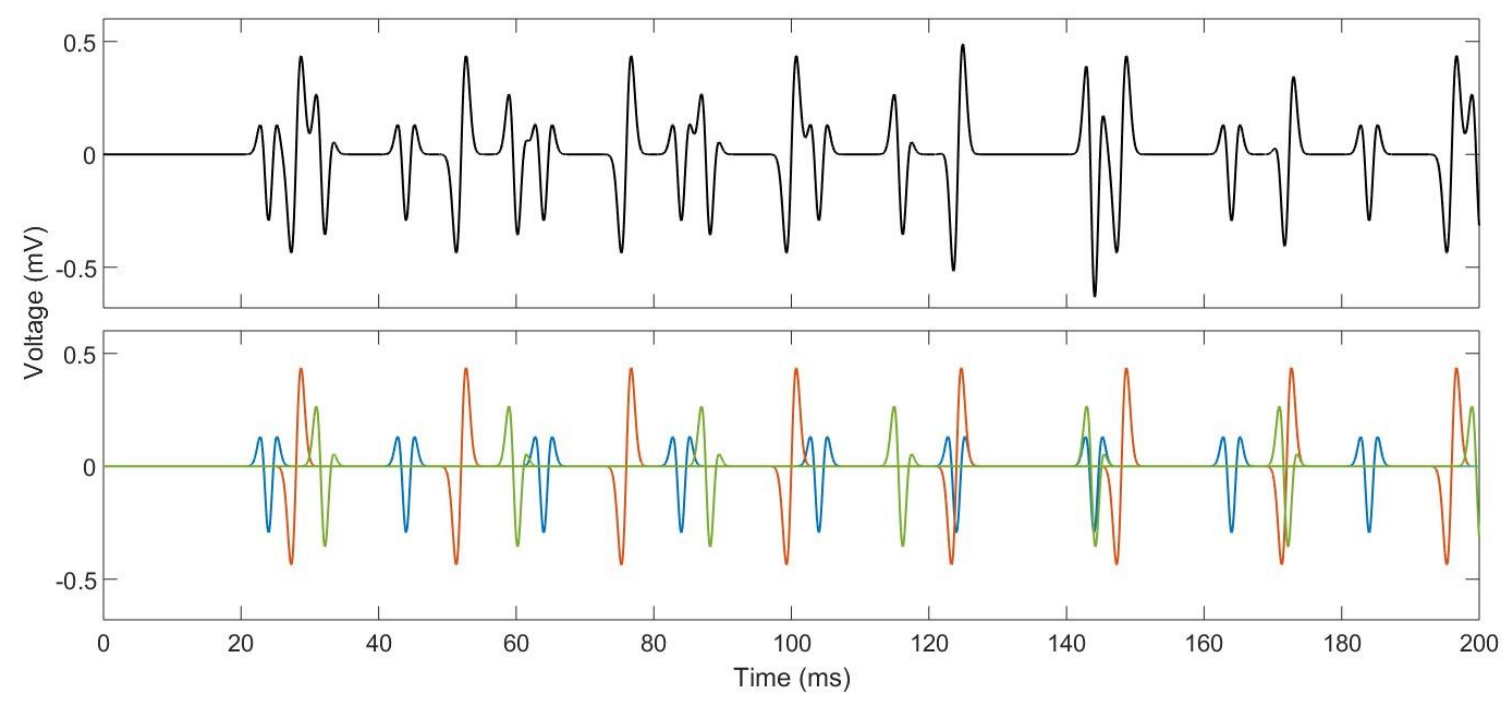

Figure 4.5: Compound synthetic sEMG signal with the three MUAPTs composing it shown below. $u_{1}$ is blue, $u_{2}$ is red, and $u_{3}$ is green.

The component functions are each of the MUAP templates time-shifted over the length of the EMG signal with spacing of $4 \mathrm{~ms}$.

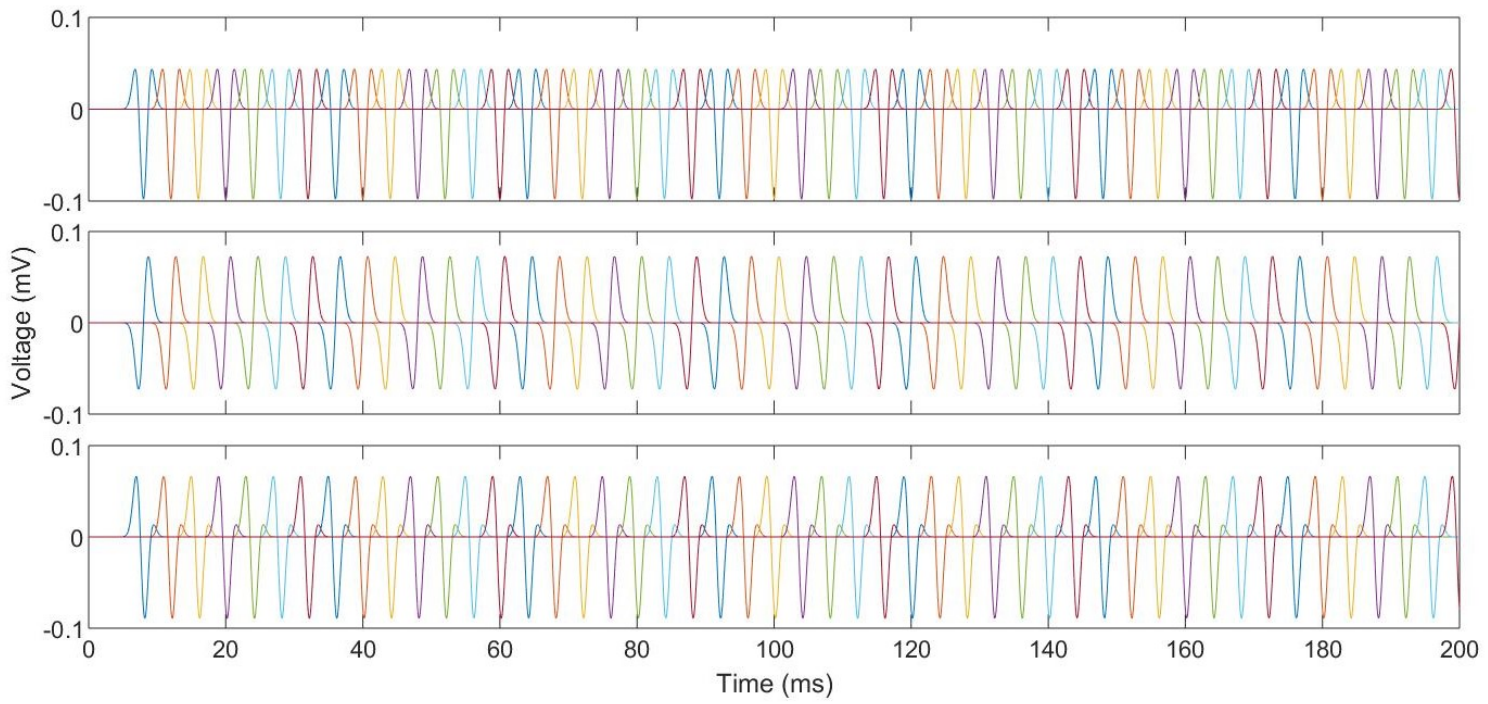

Figure 4.6: Component functions. 
The perturbation based decomposition was run on the compound sEMG function for the given MUAP component functions. Thresholding was performed separately for each MUAPT due to the dynamic range of template scaling. In Figure 4.7 below, the decomposition results before thresholding and after thresholding are displayed next to the actual constituent functions for motor unit 1.
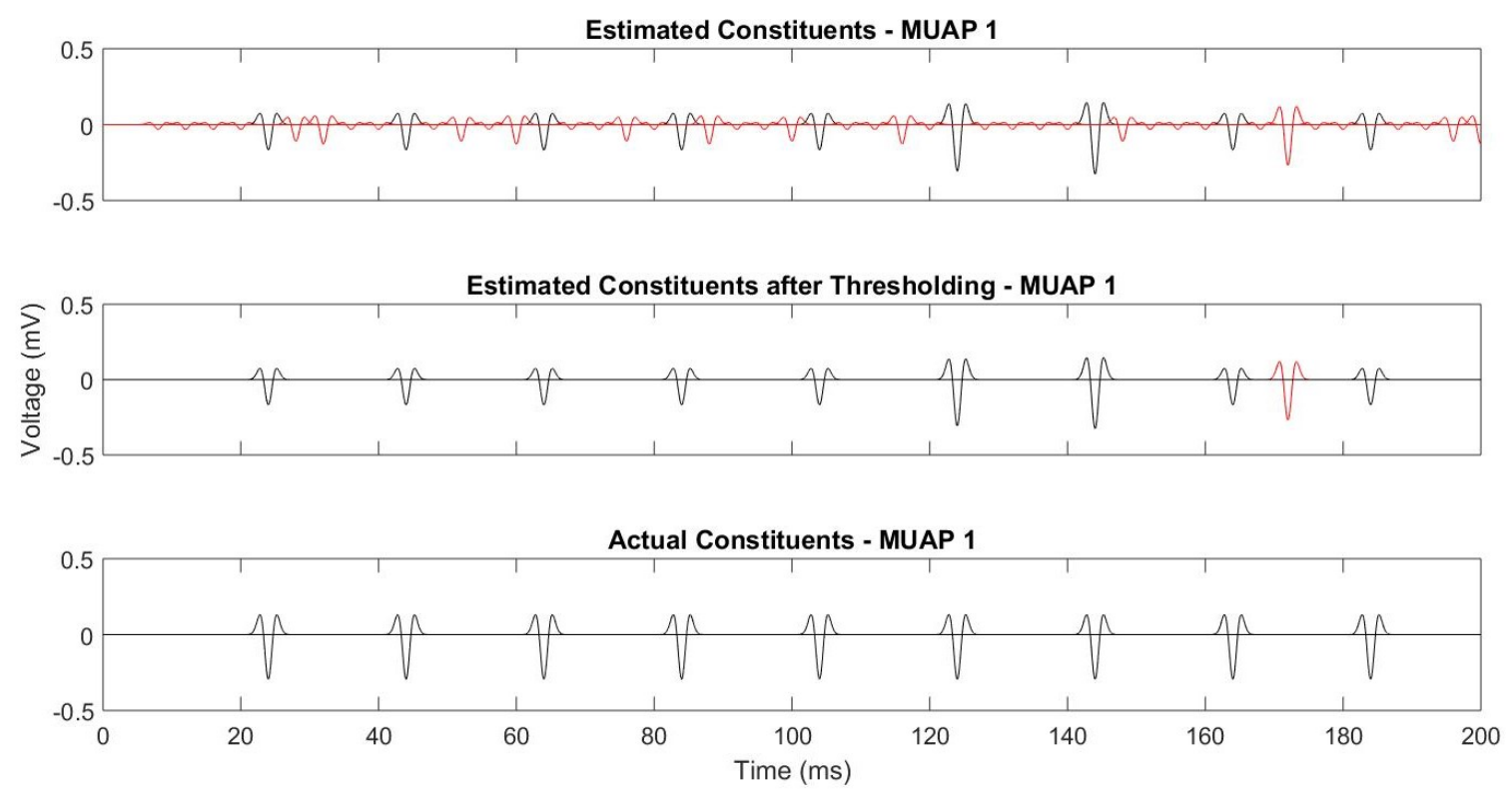

Figure 4.7: Estimated constituent functions, estimated constituent functions after thresholding $\beta$, and actual constituent functions for motor unit 1.

In Figure 4.8 below, the decomposition results before thresholding and after thresholding are displayed next to the actual constituent functions for motor unit 2 . 

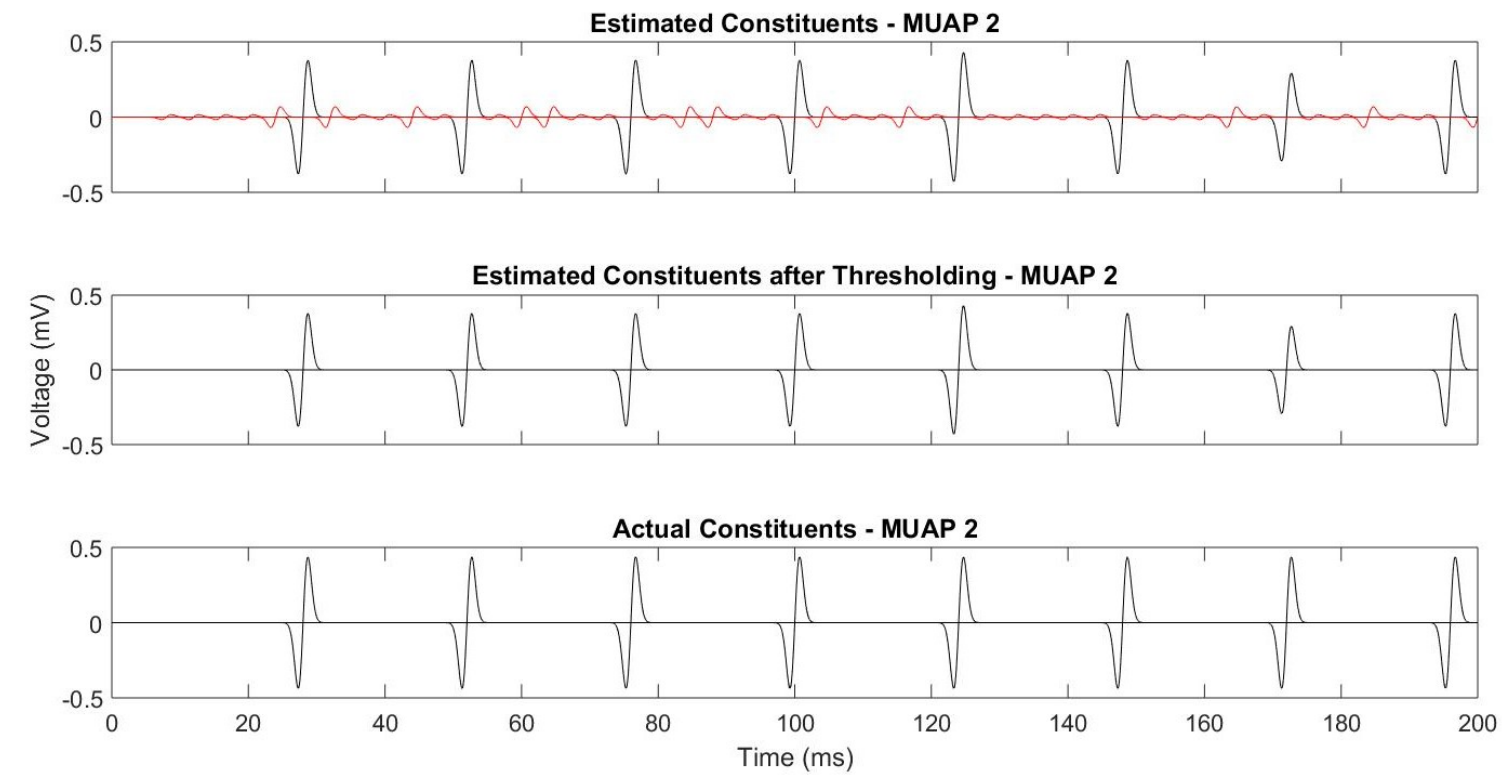

Figure 4.8: Estimated constituent functions, estimated constituent functions after thresholding $\beta$, and actual constituent functions for motor unit 2.

In Figure 4.9 below, the decomposition results before thresholding and after thresholding are displayed next to the actual constituent functions for motor unit 3. 

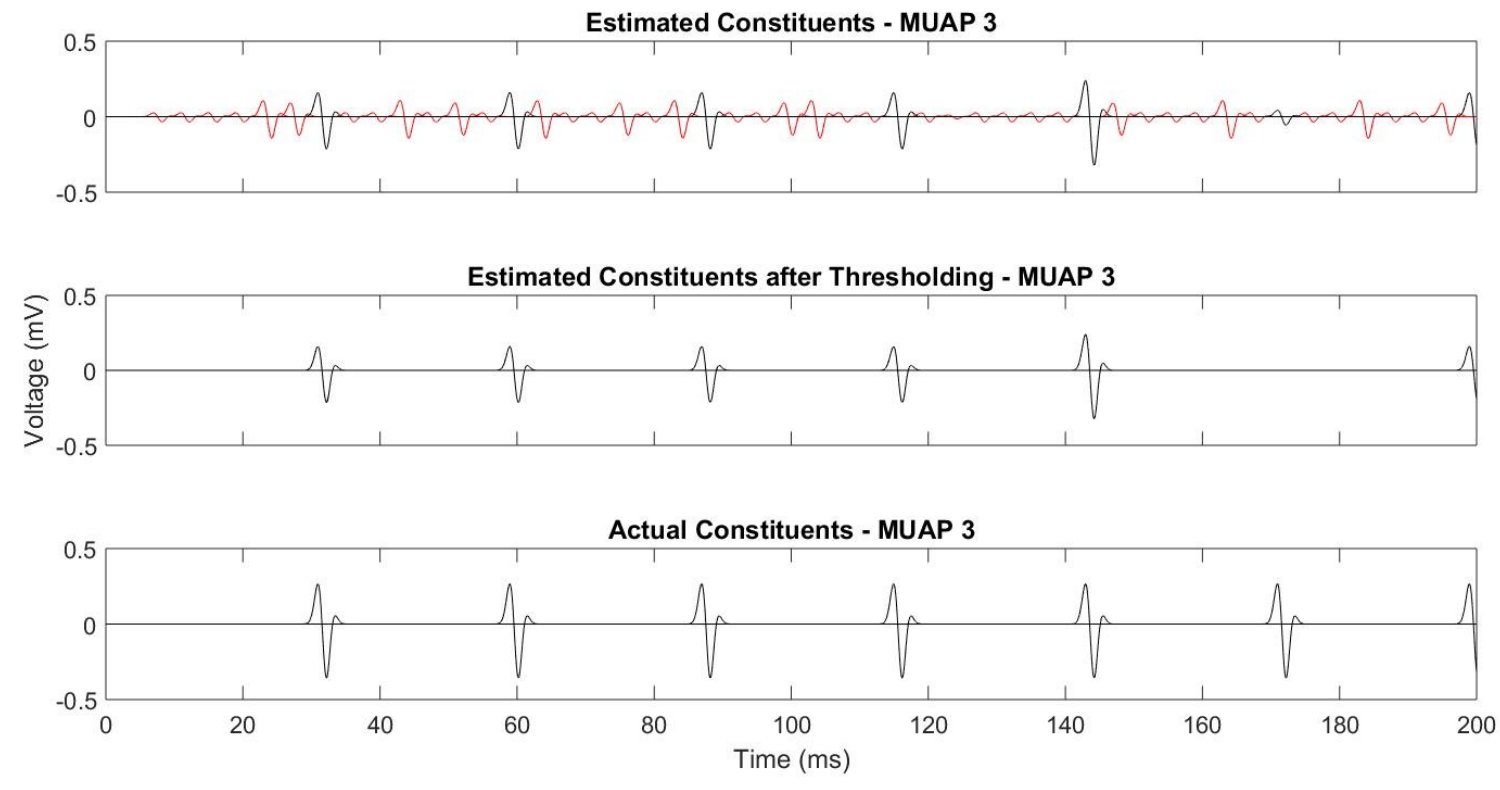

Figure 4.9: Estimated constituent functions, estimated constituent functions after thresholding $\beta$, and actual constituent functions for motor unit 3.

Figure 4.10 below displays the contribution from each component function to the compound function for the actual distribution compared with the perturbation based decomposition estimates. 


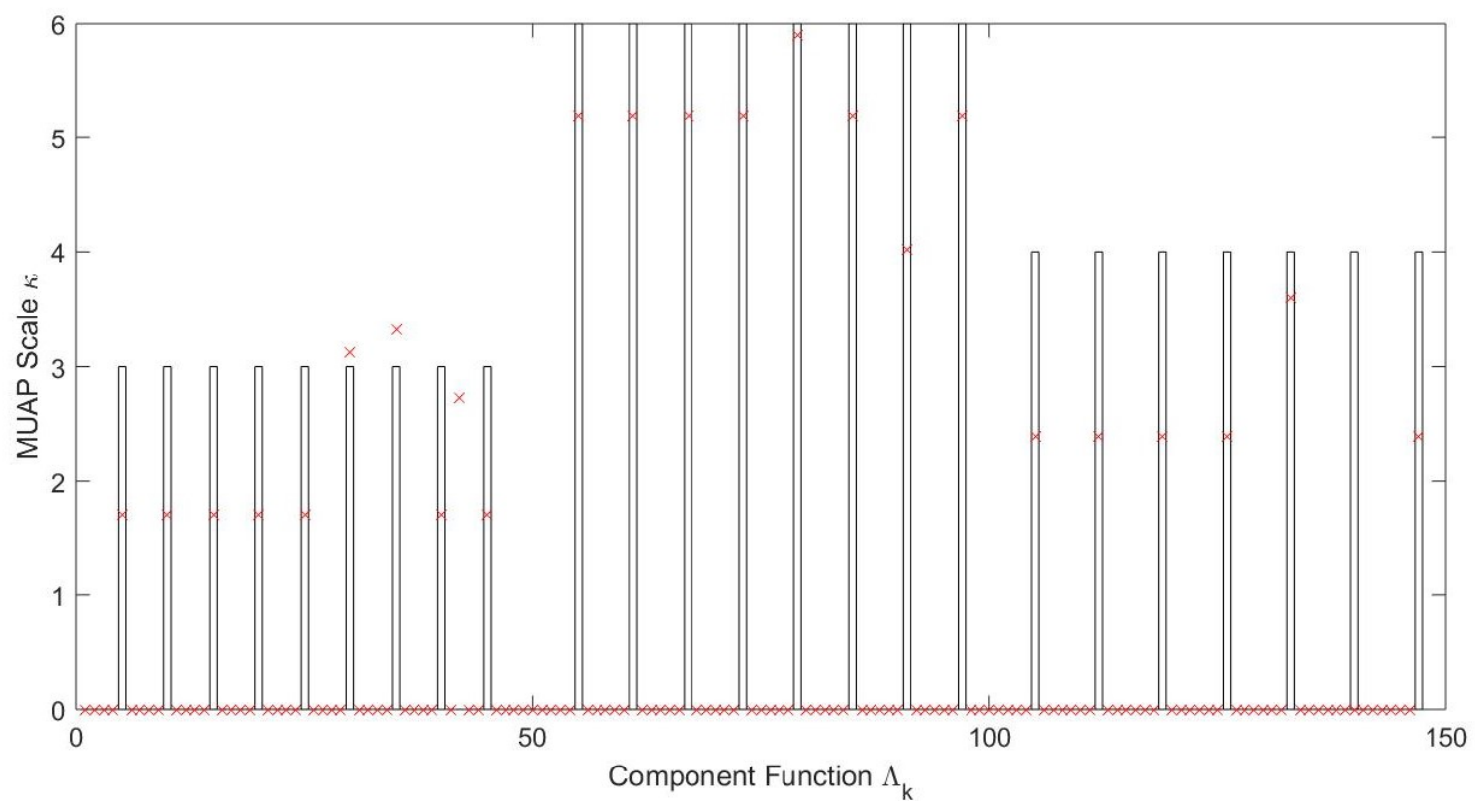

Figure 4.10: Frequency distribution of component functions after thresholding for synthetic sEMG signal.

The synthetic sEMG signal was reconstructed using the perturbation based decomposition contribution estimates after thresholding. The comparison of the sEMG signal and the reconstruction is below in Figure 4.11.

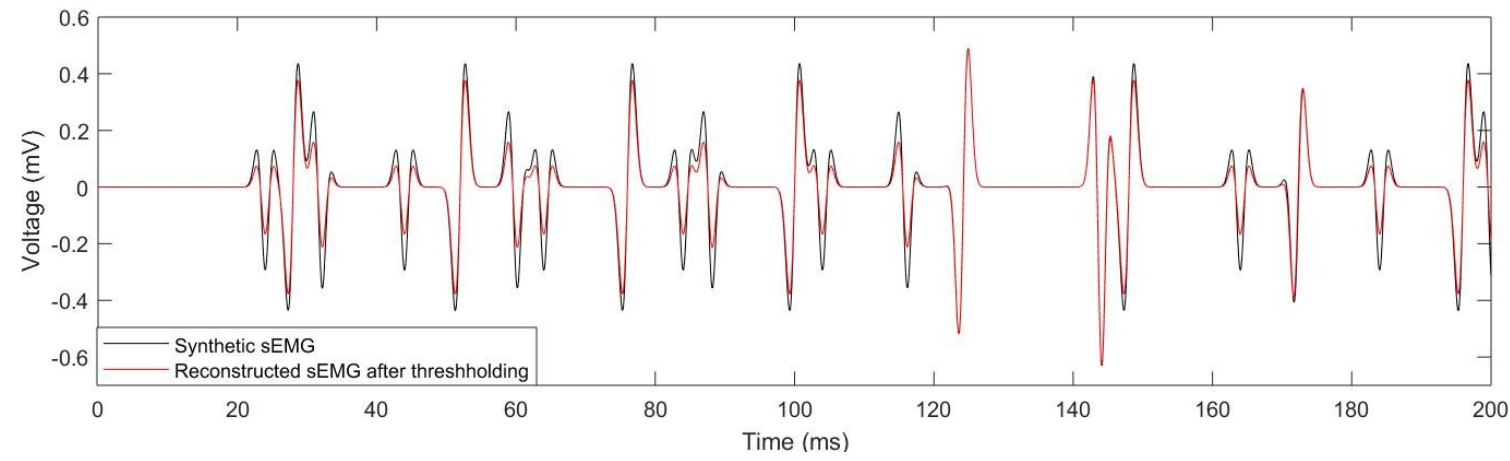

Figure 4.11: Comparison of the synthetic sEMG signal with the perturbation estimates reconstruction post-threshold. 
Table 4.2: Error table comparing actual and estimated firing rates for the MUAPTs present in the synthetic sEMG signal.

\begin{tabular}{|cccc|}
\hline MUAPT & Actual Firing Rate $(\mathrm{Hz})$ & Estimated Firing Rate $(\mathrm{Hz})$ & \% Error \\
\hline \hline$u_{1}$ & 50 & 50 & 0 \\
\hline$u_{2}$ & 41.6 & 41.6 & 0 \\
\hline$u_{3}$ & 35.7 & 35.7 & 0 \\
\hline
\end{tabular}

\subsection{Decomposition of Experimental Data}

The decomposition was tested on sEMG experimental data collected from the biceps brachii that contained six MUAPTs [1]. The MUAP template waveforms associated with each MUAPT were determined by EMGLAB and are shown in Figure 4.13. The compound sEMG function after bandpass filtering from $10 \mathrm{~Hz}$ to $1 \mathrm{kHz}$ is shown in Figure 4.12 .

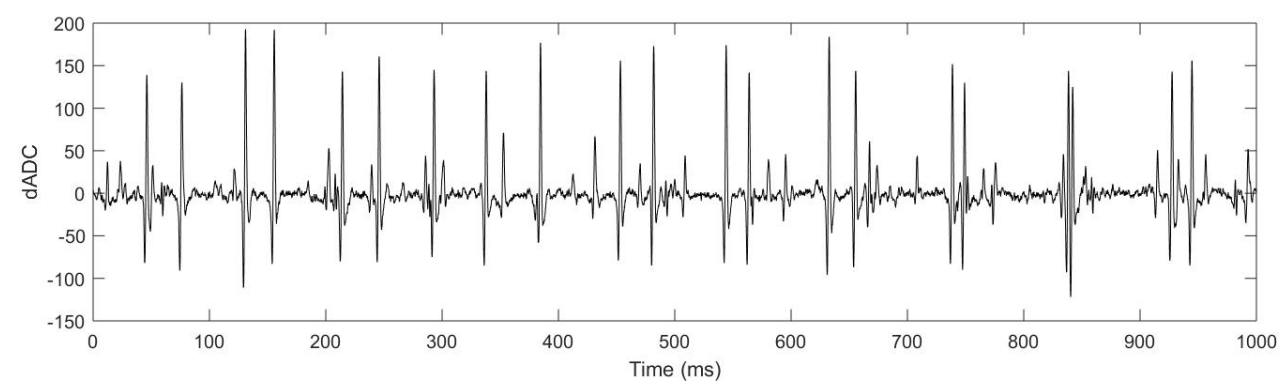

Figure 4.12: sEMG signal from biceps brachii. 

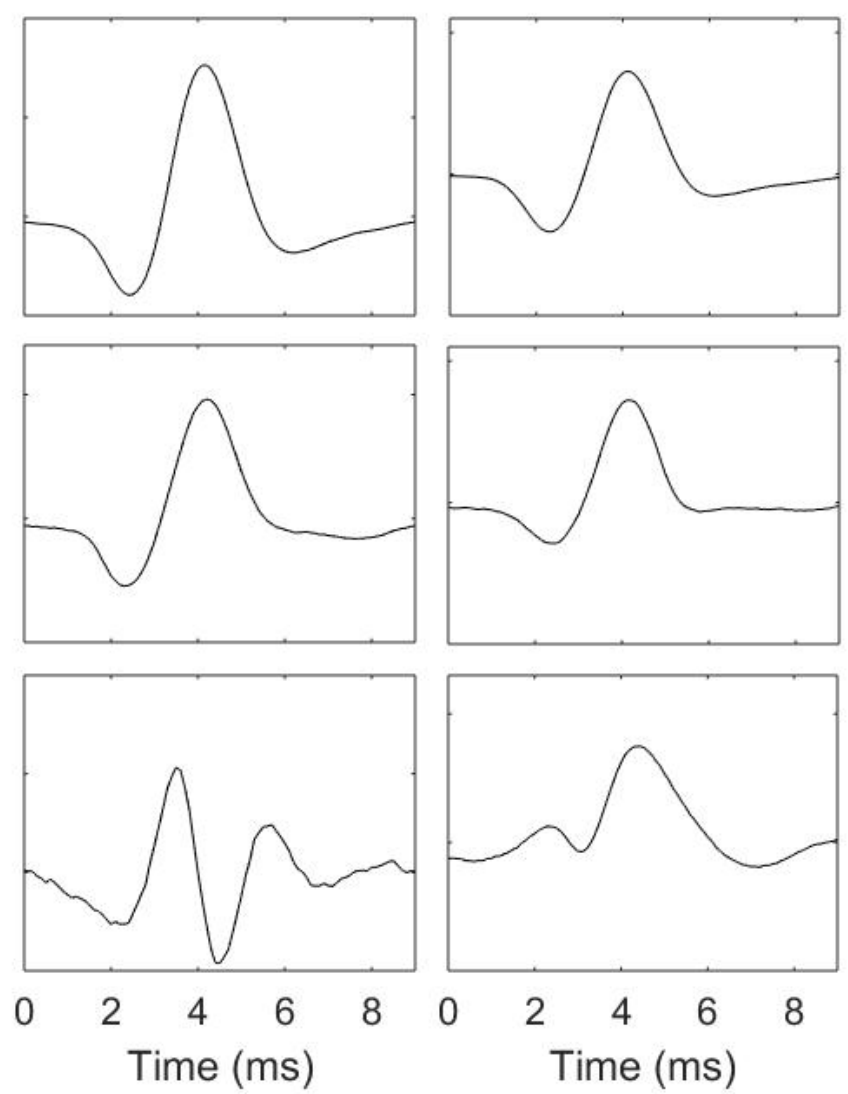

Figure 4.13: Templates for MUAP shapes present in sEMG signal.

EMGLAB decomposed the MUAPTs using an algorithm followed by considerable manual editing. The EMGLAB decomposition results for one of the MUAP template shapes over the length of the entire sEMG (1000ms) is displayed in Figure 4.14. The results of the perturbation based decomposition will be compared with EMGLAB's decomposition for the six MUAP template shapes. 


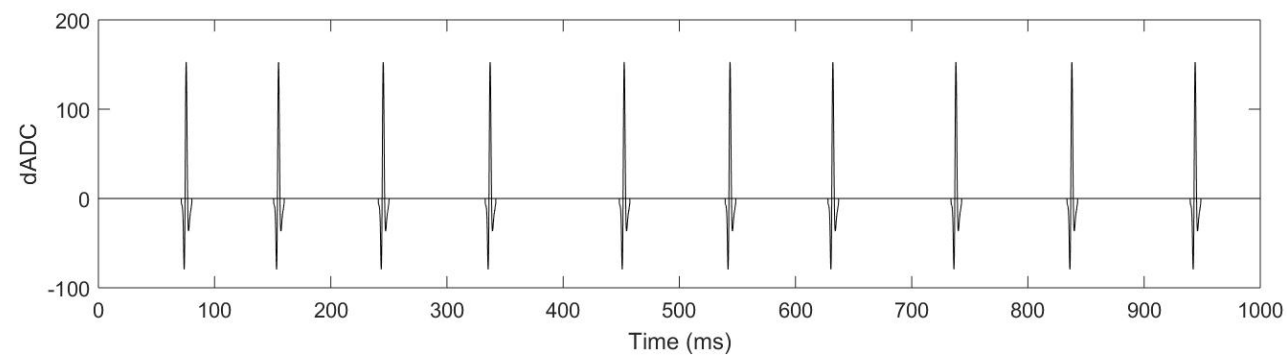

Figure 4.14: EMGLAB decomposition results shown for a single MUAP template shape present in the sEMG in Figure 4.12 .

The component functions used for the perturbative approximate series expansion were the six MUAP template shapes time-shifted over the length of the decomposition region of the sEMG signal with a spacing of $2.5 \mathrm{~ms}$. Due to system constraints, only small sections of the sEMG signal could be decomposed at a time, with the success of the decomposition highly dependent on the selection of the section of sEMG signal. Usually there were zero to two firings of each MUAP template per decomposition section.

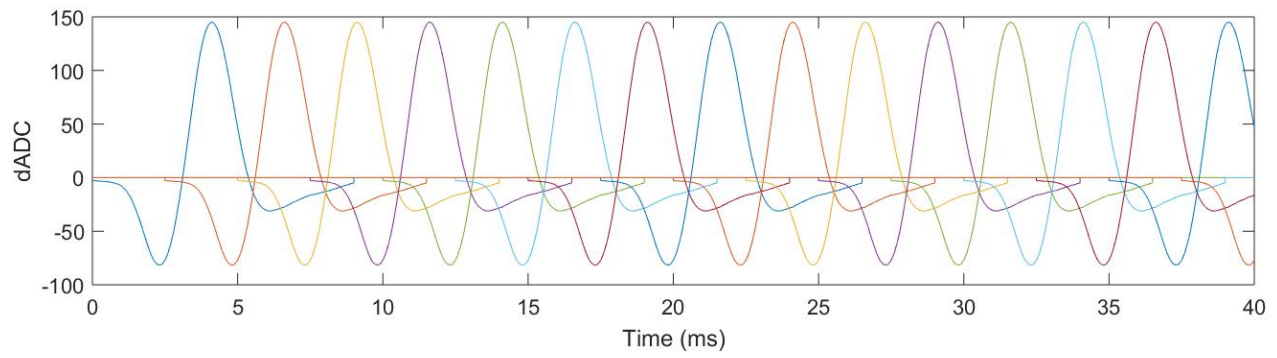

Figure 4.15: Representative component functions for a single MUAP template shape with shifts of $2.5 \mathrm{~ms}$.

The below example of the decomposition of $40 \mathrm{~ms}$ of sEMG signal is representative of the overall accuracy of the technique. In the 40ms section of sEMG signal, four of the MUAP templates each fired one time while the other two MUAP temples did not fire at all (according to EMGLAB decomposition).

In Figure 4.16 below, the contribution estimates for the MUAP 2 component func- 
tions improve greatly with thresholding. After thresholding, there are two MUAPs identified instead of one.
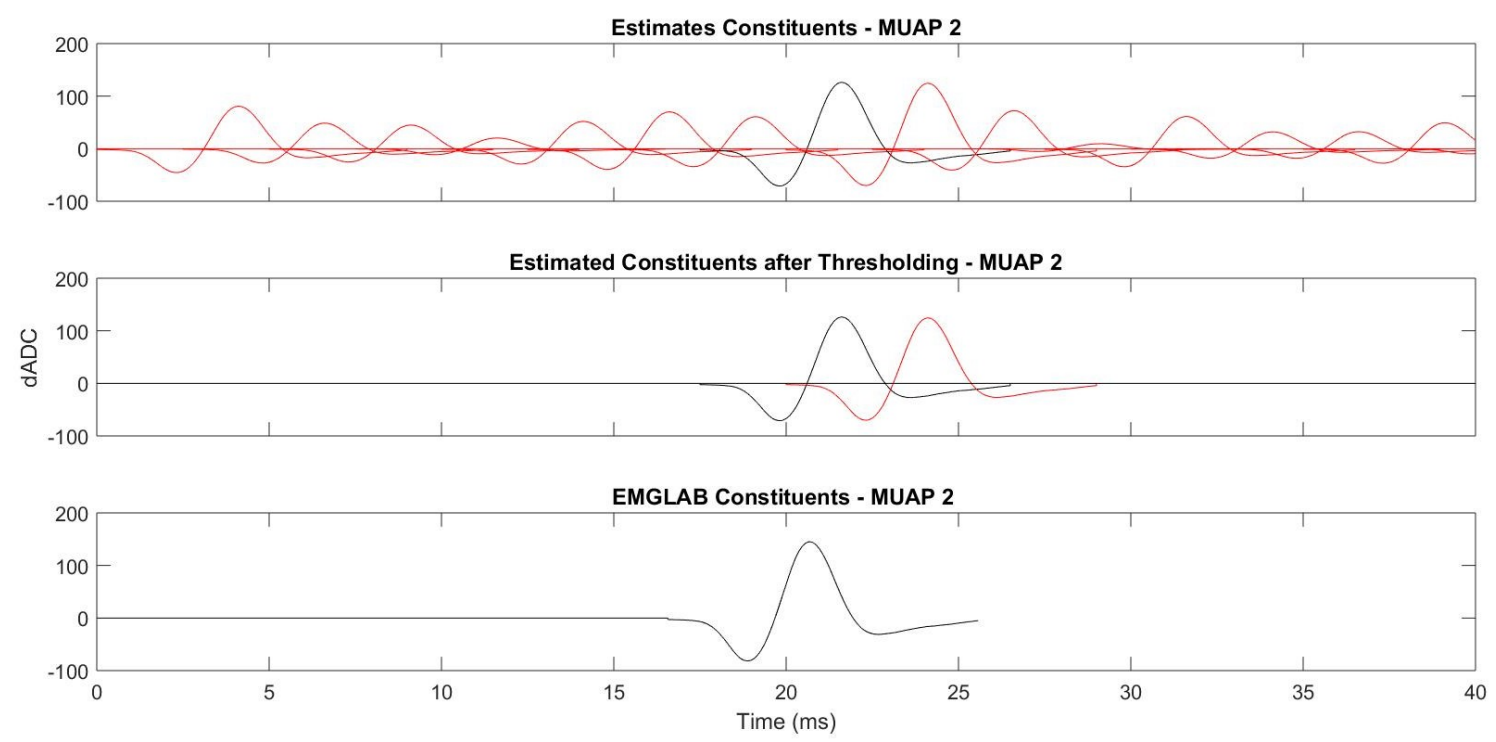

Figure 4.16: Estimated constituent functions, estimated constituent functions after thresholding, and actual constituent functions for motor unit 2 .

In Figure 4.17 below, the contribution estimates for the MUAP 3 component functions improve greatly with thresholding. After thresholding, there are two MUAPs identified instead of one. 

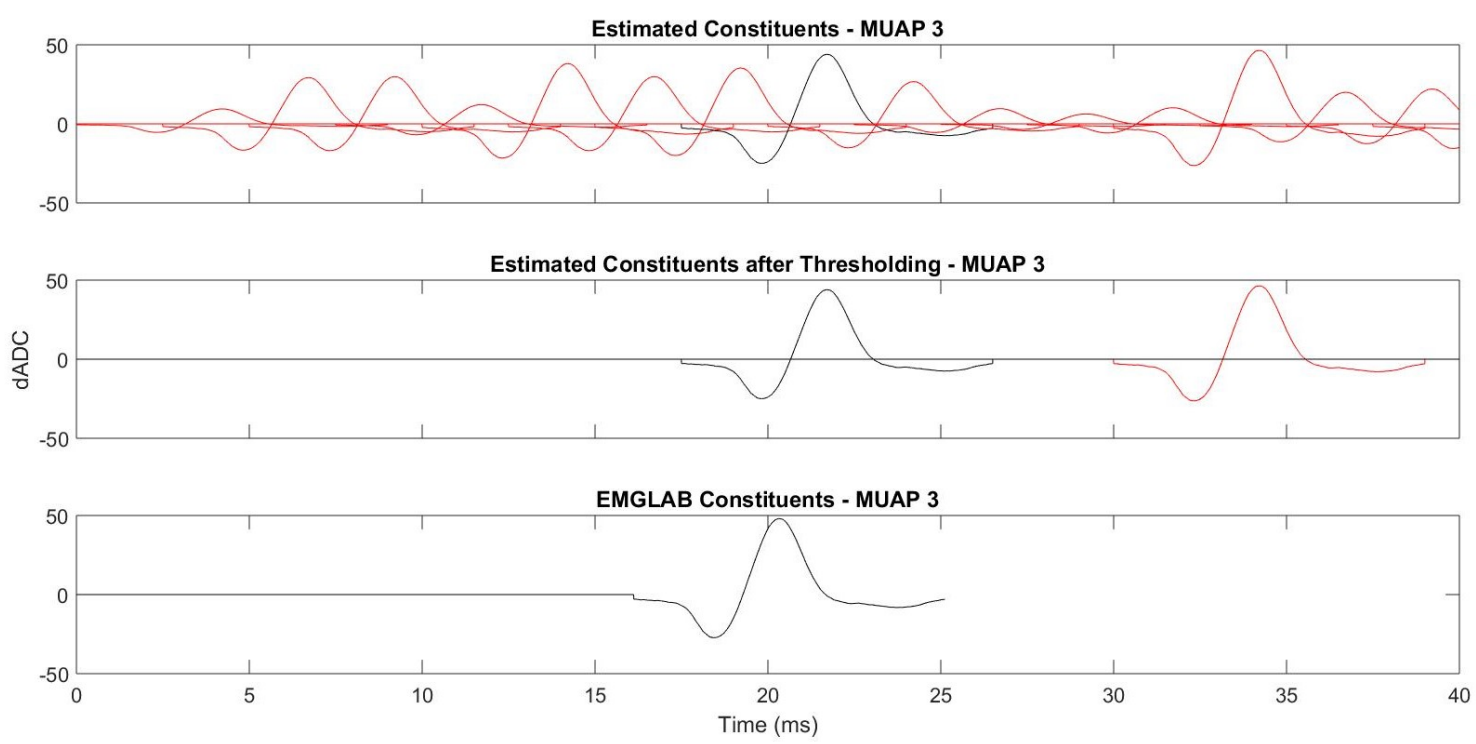

Figure 4.17: Estimated constituent functions, estimated constituent functions after thresholding, and actual constituent functions for motor unit 3.

In Figure 4.18 below, the contribution estimates for the MUAP 5 component functions improve greatly with thresholding. After thresholding, there are two MUAPs identified instead of one. 

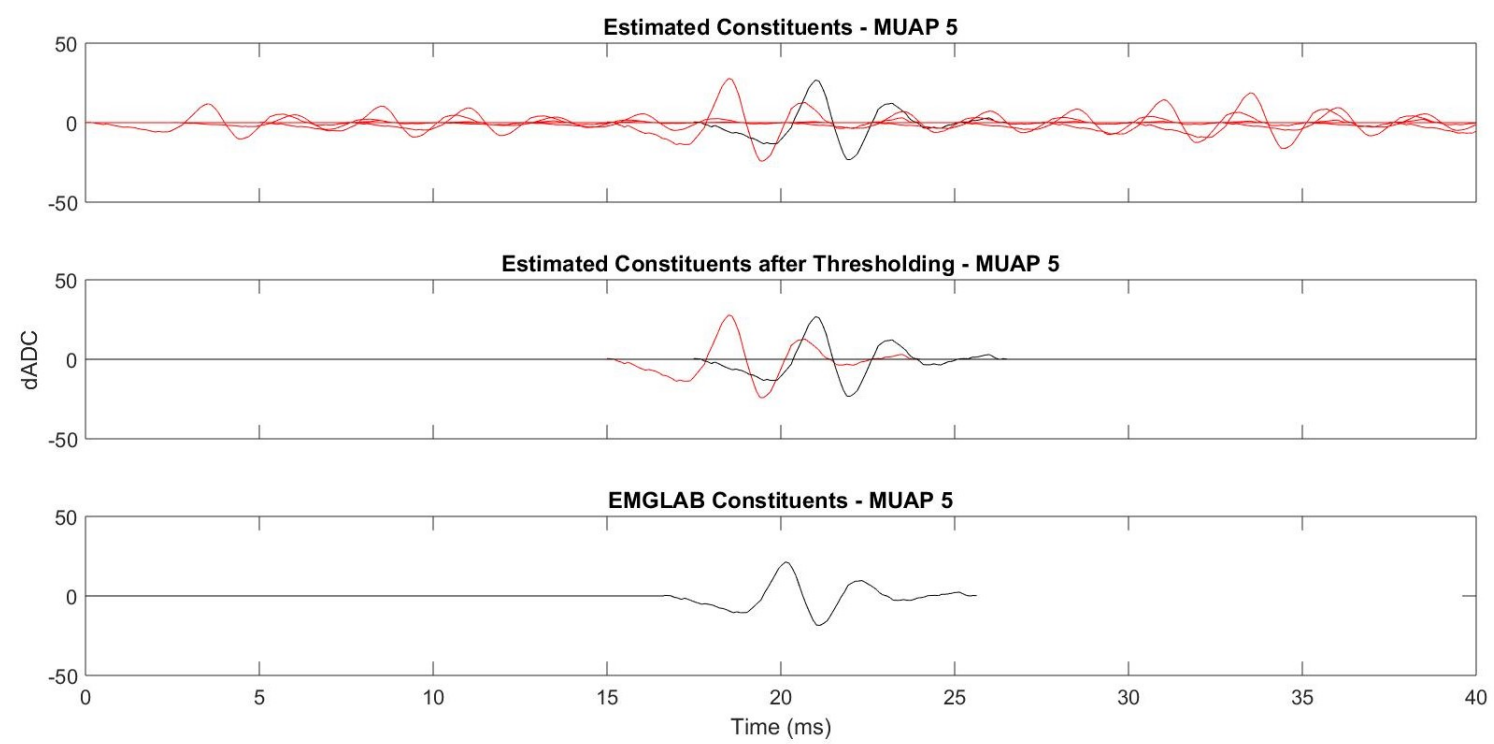

Figure 4.18: Estimated constituent functions, estimated constituent functions after thresholding, and actual constituent functions for motor unit 5.

In Figure 4.19 below, the contribution estimates for the MUAP 6 component functions improve greatly with thresholding. After thresholding, there are three MUAPs identified instead of one. 

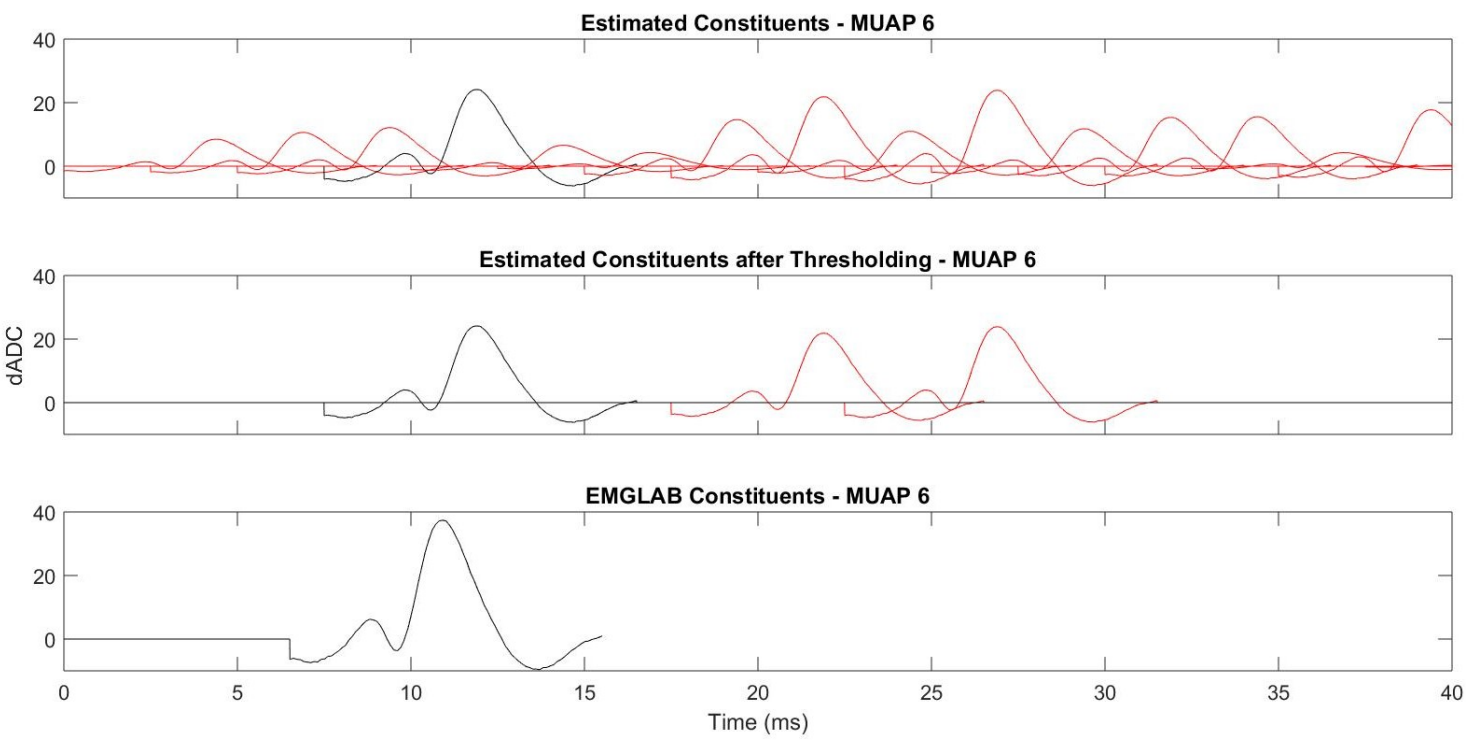

Figure 4.19: Estimated constituent functions, estimated constituent functions after thresholding, and actual constituent functions for motor unit 6. 


\section{Discussion}

This work explored the feasibility of using Szlavik's perturbative approximate series expansion method for analysis of sEMG signals. While successful for synthetic signals, the decomposition worked inconsistently for experimental EMG signals for the following reasons:

- Spacing of component functions:

For experimental sEMG signals, the firing times of MUAPs are analog, while the component functions used as inputs to the perturbation based decomposition have discrete firing times. This causes issues when the experimental MUAP has a firing time in between two component MUAPs firing times. The decomposition commonly estimates the presence of two MUAPs instead of one, as in Figure 4.18.

- Similarity of MUAP waveforms:

Similar MUAP shapes between different motor units causes difficulty for the algorithm as may it misidentify an action potential, or estimate both of the similar MUAP component functions as contributors.

- Complexity of sEMG signal:

Signals with larger numbers of motor units, larger ranges in amplitude sizes between MUAPs, and more similar MUAP shapes increase complexity of sEMG signal and the difficulty of decomposition. 


\subsection{Implications}

Successful decomposition of sEMG signals, especially for firing times of MUAPs, is useful for evaluation of neuromuscular diseases as well as in clinical research. There is a need for fast, accurate, and easy-to-use EMG signal decomposition for better understanding of normal muscle activation, as well as muscle activation affected by disease states, fatigue, aging, space, and pharmaceuticals [9]. The noninvasive nature of sEMG allows for less experienced operators to record signals with less pain and risk to subjects, but it comes with increased decomposition challenges. The perturbative expansion series method's ability to handle nonorthogonal component functions with limited user intervention (only manual thresholding) makes it a good candidate for sEMG signal decomposition. The success of the decomposition with both synthetic and experimental signals shows promise for improved ease of decomposition for diagnosis as well as research. The decomposition occasionally missed MUAPs or misidentified MUAPs present in the signal. The decomposition was more accurate at identifying the timing of MUAPs than at determining amplitude of MUAPs.

\subsection{Limitations and Future Work}

The major limitations affecting success of sEMG decomposition were similarity in shape of action potential waveforms from the different motor units composing the EMG signal and the high degrees of variability and complexity between sEMG signals. Future work may involve setting the component functions as the main MUAP template shapes present throughout sEMG signals, with a further decomposition algorithm applied after the perturbation based decomposition to determine MUAPTs of each template type (using the assumptions of constant firing rates and constant amplitudes within a MUAPT). Additionally, incorporating knowledge of the refractory 
period into the sEMG perturbation based decomposition technique would improve the technique for cases in which adjacent component functions within a motor unit template are both estimated to fire.

Other important limitations were computing power and memory. Variables affecting the required computing power and memory for decomposition were the length of the EMG signal, number of component functions (smaller spacing between component functions increases accuracy of the decomposition while requiring significantly more memory), and the sampling rate of the EMG signal.

An additional limitation was the requirement for the system of component functions used in decomposition of the compound function to remain overdetermined. For the system to be overdetermined, the number of time points in the compound signal must be greater than the component functions squared minus the number of component functions. As the number of MUAP templates used as inputs to the decomposition increases, and thus the number of component functions increases, the number of timepoints in the compound signal must be increased to satisfy the inequality. For the experimental data sampled at $10 \mathrm{kHz}$, the compound EMG signal time-points had to be interpolated by a factor of up to thirty (depending on the length of the sEMG segment and component function spacing) to solve the decomposition which further increased the required computing power and memory.

\subsection{Conclusion}

The ability to detect small changes in muscle activation without insertion of needle electrodes or requirement for a well-trained operator would greatly increase the applications for EMG in nonclinical research, clinical research, and diagnosis of neuromuscular disorders. Accessible, convenient, and easy-to-use sEMG decomposition 
(with minimal user intervention) would allow for more research into muscle activation and a better understanding of neurodegenerative disorders. 


\section{BIBLIOGRAPHY}

[1] "J-Y Hogrel." [Online dataset R011, available at http://www. emglab.net].

[2] "Cal Poly Github." http://www.github.com/CalPoly.

[3] L. Biga, S. Dawson, A. Harwell, R. Hopkins, J. Kaufmann, M. LeMaster, P. Matern, K. Morrison-Graham, D. Quick, J. Runyeon, and L. Whittier, Anatomy Physiology. Open Oregon State, Oregon State University, 2018.

[4] R. Thornton and C. Michell, "Techniques and applications of EMG: Measuring motor units from structure to function," Journal of Neurology, vol. 259, no. 3, pp. 585-594, 2012.

[5] X. Ren, C. Zhang, X. Li, G. Yang, T. Potter, and Y. Zhang, "Intramuscular EMG decomposition basing on motor unit action potentials detection and superposition resolution. frontiers in neurology," Frontiers in Neurology, vol. 9, no. 2, 2018.

[6] P. Zhou and W. Rymer, "Muap number estimates in surface EMG:

Template-matching methods and their performance boundaries," Annals of Biomedical Engineering, vol. 32, no. 7, pp. 1007-1015, 2004.

[7] C. D. Luca, A. Adam, R. Wotiz, L. Gilmore, and S. Nawab, "Decomposition of surface EMG signals," Journal of Neurophysiology, vol. 96, no. 3, pp. 1646-1657, 2006.

[8] T. Masuda, H. Miyano, and T. Sadoyama, "A surface sensor array for detecting action potential trains of single motor units," Electroencephalography and Clinical Neurophysiology, vol. 60, pp. 443-453, 1985. 
[9] S. Nawab and C. D. Luca, "Decomposition of indwelling emg signals.," Journal of Applied Physiology, vol. 105, no. 2, pp. 700-710, 2008.

[10] B. Klein, J. Blok, R. Oostenveld, P. Praamstra, and D. Stegeman, "Magnetic stimulation-induced modulations of motor unit firings extracted from multi-channel surface emg.," Muscle Nerve, vol. 23, pp. 1000-1015, 2000.

[11] A. Holobar and D. Zazula, "Correlation-based decomposition of surface electromyograms at low contraction forces," Medical Biological Engineering Computing, vol. 42, pp. 487-495, 2004.

[12] C. Katsis, Y. Goletsis, A. Likas, D. Fotiadis, and I. Sarmas, "A novel method for automated EMG decomposition and muap classification," Artificial Intelligence in Medicine., vol. 37, no. 1, pp. 55-64, 2006.

[13] G. Garcia, R. Okuno, and K. Akazawa, "Technological developments in japan - a decomposition algorithm for surface electrode-array electromyogram: a noninvasive, three-step approach to analyze surface emg signals.," Engineering in Medicine and Biology Magazine, vol. 24, pp. 65-72, 2005.

[14] M. Gazzoni, D. Farina, and R. Merletti, "A new method for the extraction and classification of single motor unit action potentials from surface emg signals.," Journal of Neuroscience Methods, vol. 136, pp. 165-177, 2004.

[15] G. Biagetti, P. Crippa, S. Orcioni, and C. Turchetti, "Homomorphic deconvolution for muap estimation from surface EMG signals," IEEE Journal of Biomedical and Health Informatics, vol. 21, no. 2, pp. 328-338, 2016.

[16] M. Barbero, R. Merletti, and A. Rainoldi, Atlas of Muscle Innervation Zones: 
Understanding Surface Electromyography and Its Applications. Springer Milan, 2012.

[17] R. Merletti and C. D. Luca, "New techniques in surface electromyography.," Computer Aided Electromyography and Expert Systems., vol. 9, no. 3, pp. 115-124, 1989.

[18] D. Stashuk, "EMG signal decomposition: How can it be accomplished and used?," Journal of Electromyography and Kinesiology, vol. 11, no. 3, pp. 151-173, 2001.

[19] M. Raez, M. Hussain, and F. Mohd-Yasin, "Techniques of EMG signal analysis: Detection, processing, classification and applications (correction)," Biological Procedures Online, vol. 8, no. 1, p. 163, 2006.

[20] J. Halonen, P. Falck, and B. Kalimo, "The firing rate of motor units in neuromuscular disorders.," Journal of Neurology, vol. 225, no. 4, pp. 269-276, 1981.

[21] B. Mokhlesabadifarahani and V. Gunjan, EMG Signals Characterization in Three States of Contraction by Fuzzy Network and Feature Extraction. SpringerBriefs in Applied Sciences and Technology, 2015.

[22] K. McGill, Z. Lateva, and H. Marateb, "Emglab: an interactive emg decomposition program.," Journal of Neuroscience Methods, vol. 149, no. 2, pp. 121-133, 2005. [The software is available at http://www.emglab.net].

[23] R. Szlavik, "A perturbation based decomposition of compound-evoked potentials for characterization of nerve fiber size distributions.," IEEE Transactions on Neural Systems and Rehabilitation Engineering, vol. 24, no. 2, pp. 212-216, 2016. 
[24] S. Jue and R. Szlavik, "Characterizing neurotransmitter receptor activation with a perturbation based decomposition method.," 2016.

[25] R. Szlavik, D. Paquin, and G. E. T. III, "A perturbative-based generalized series expansion in terms of non-orthogonal component functions," Applied Mathematics, vol. 8, pp. 106-116, 2017.

[26] R. Chowdhury, M. Reaz, M. Mohd, A. Bakar, K. Chellappan, and T. Chang, "Surface electromyography signal processing and classification techniques.," Sensors, vol. 13, no. 9, pp. 12431-12466, 2013.

[27] Y. Ning and Y. Zhang, "A new approach for multi-channel surface emg signal simulation.," Biomedical Engineering Letters, vol. 7, no. 1, pp. 45-53, 2017. 


\section{APPENDIX A Synthetic MUAP Model}

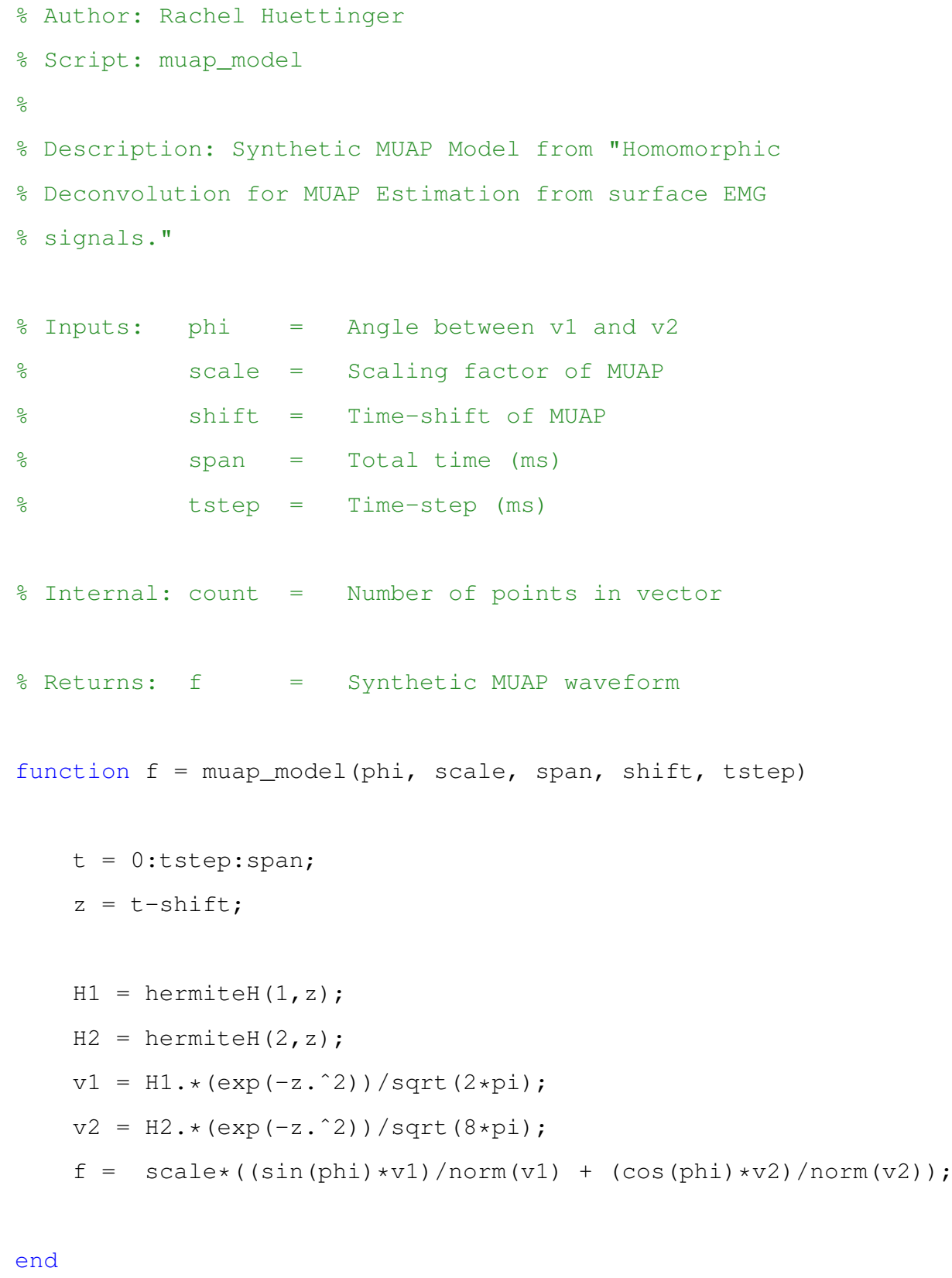




\section{B Synchronous MUAP Decomposition}

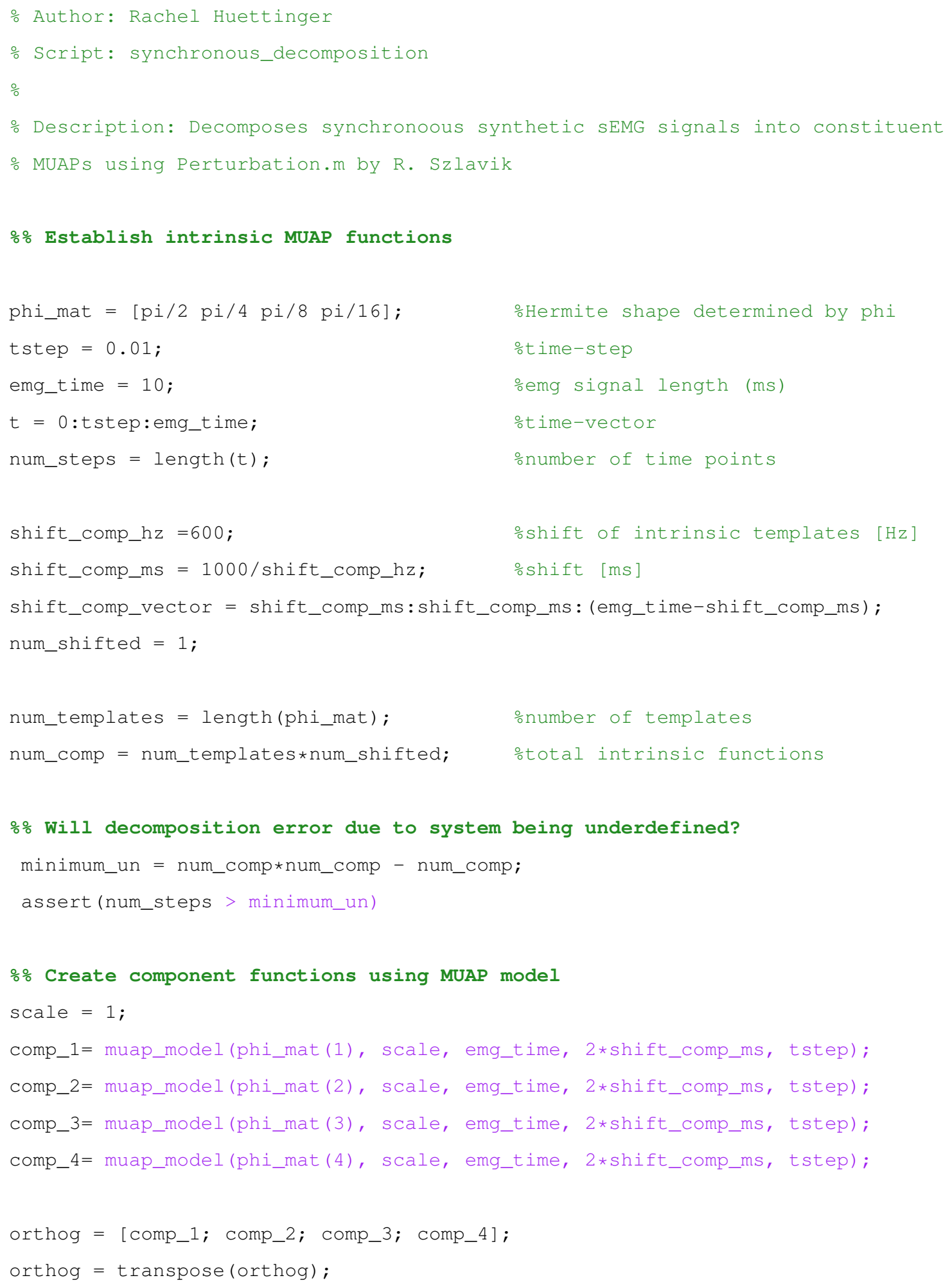




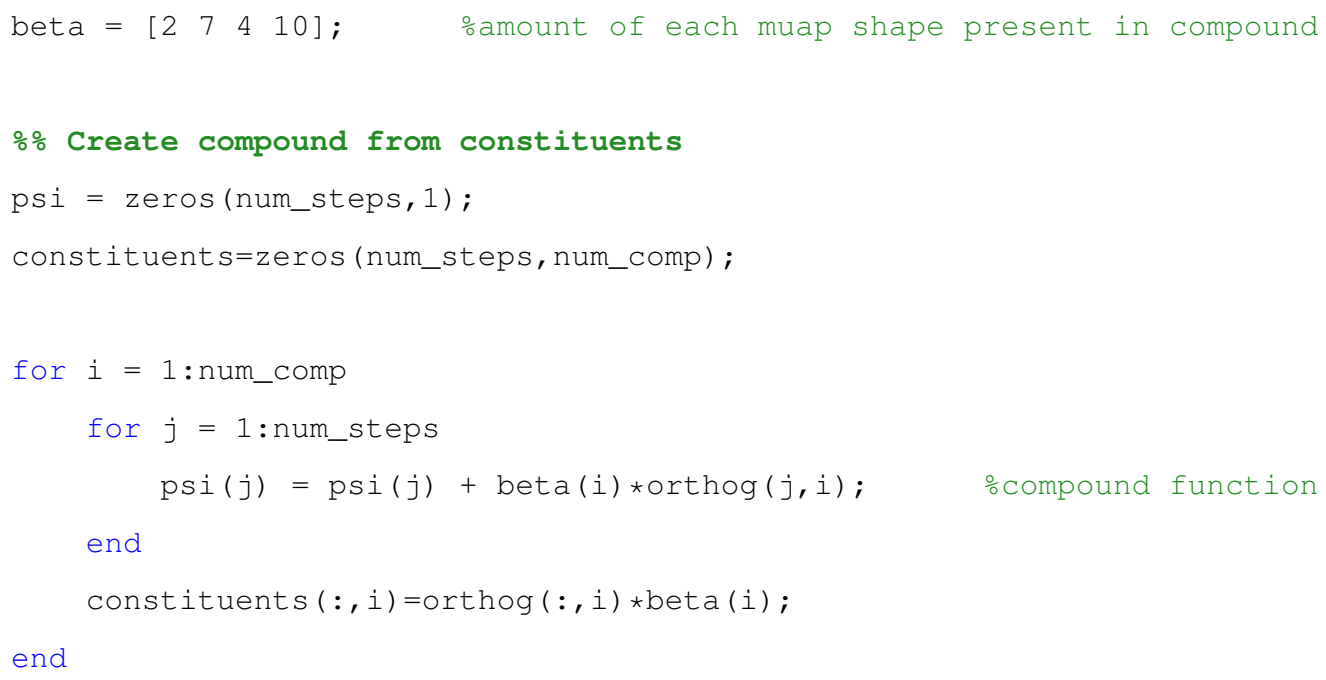




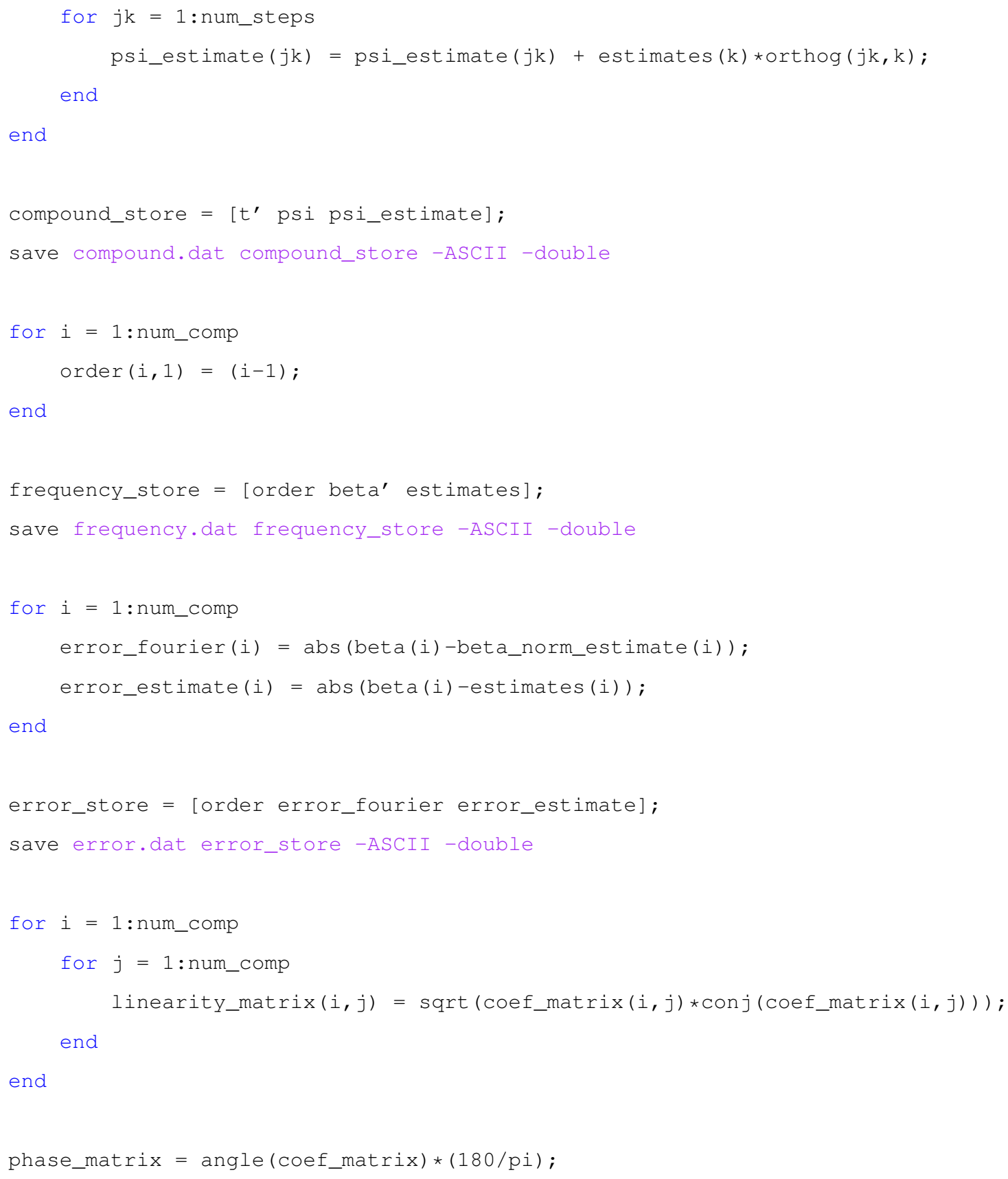




\section{Synthetic sEMG MUAP Decomposition}

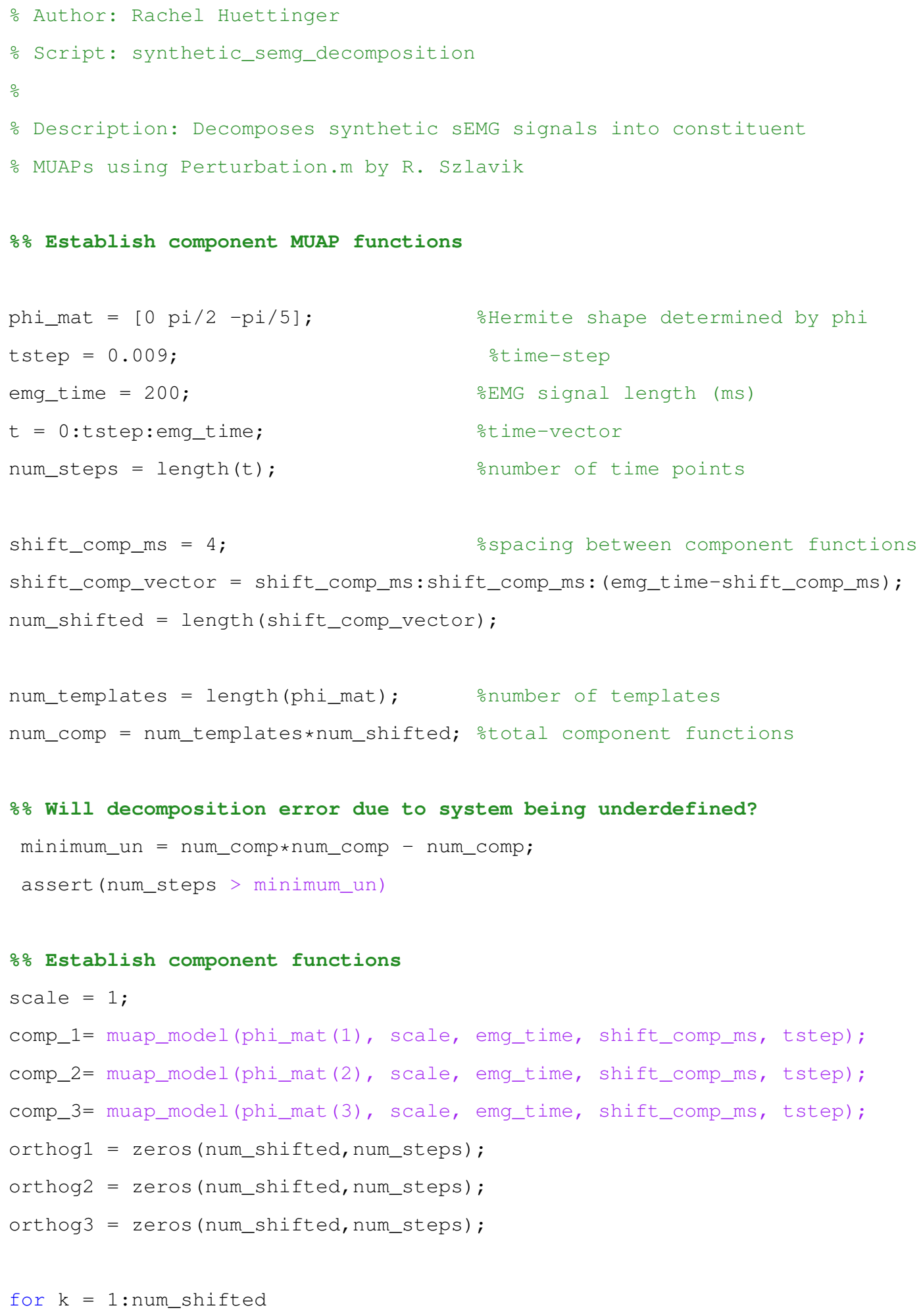




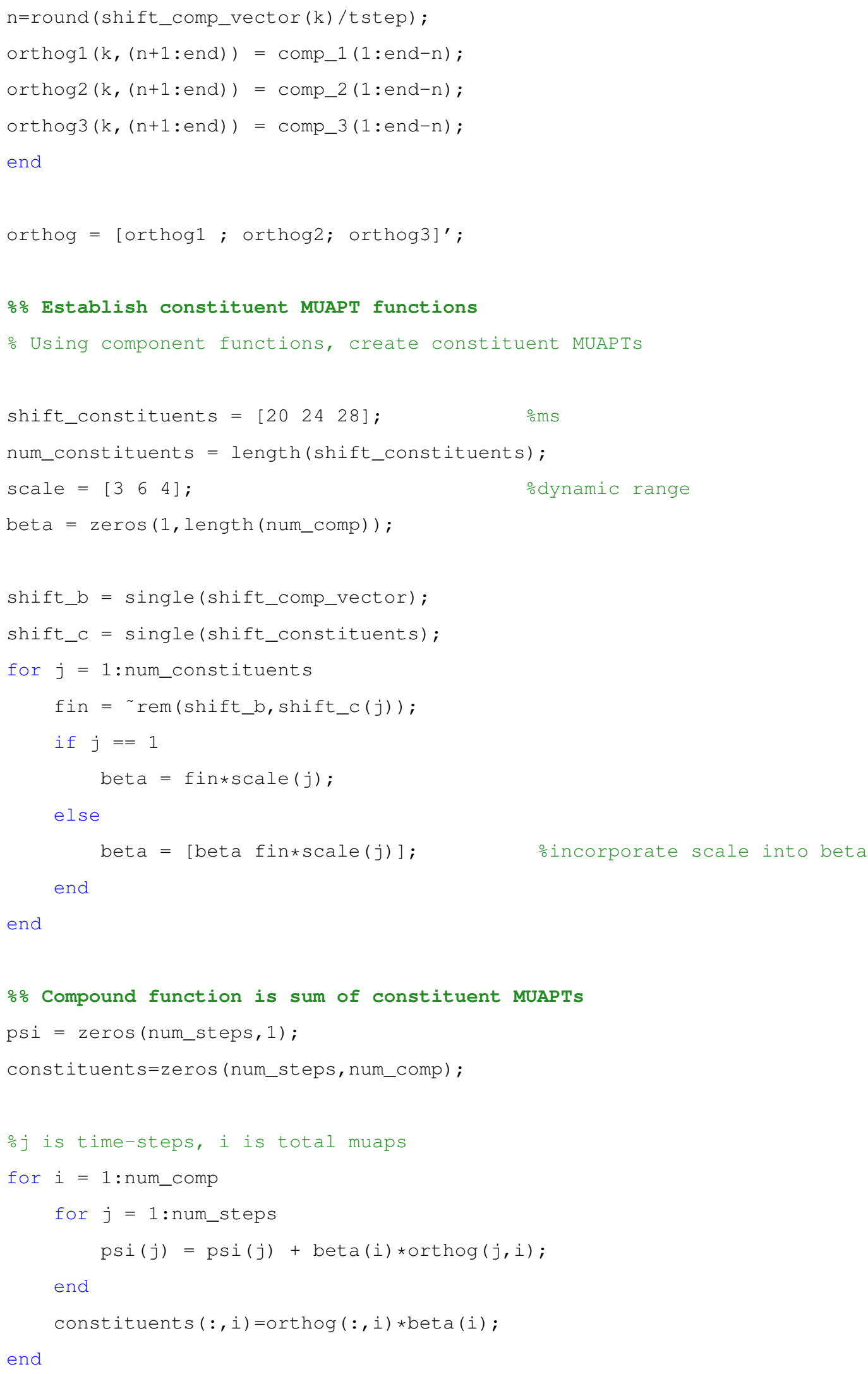




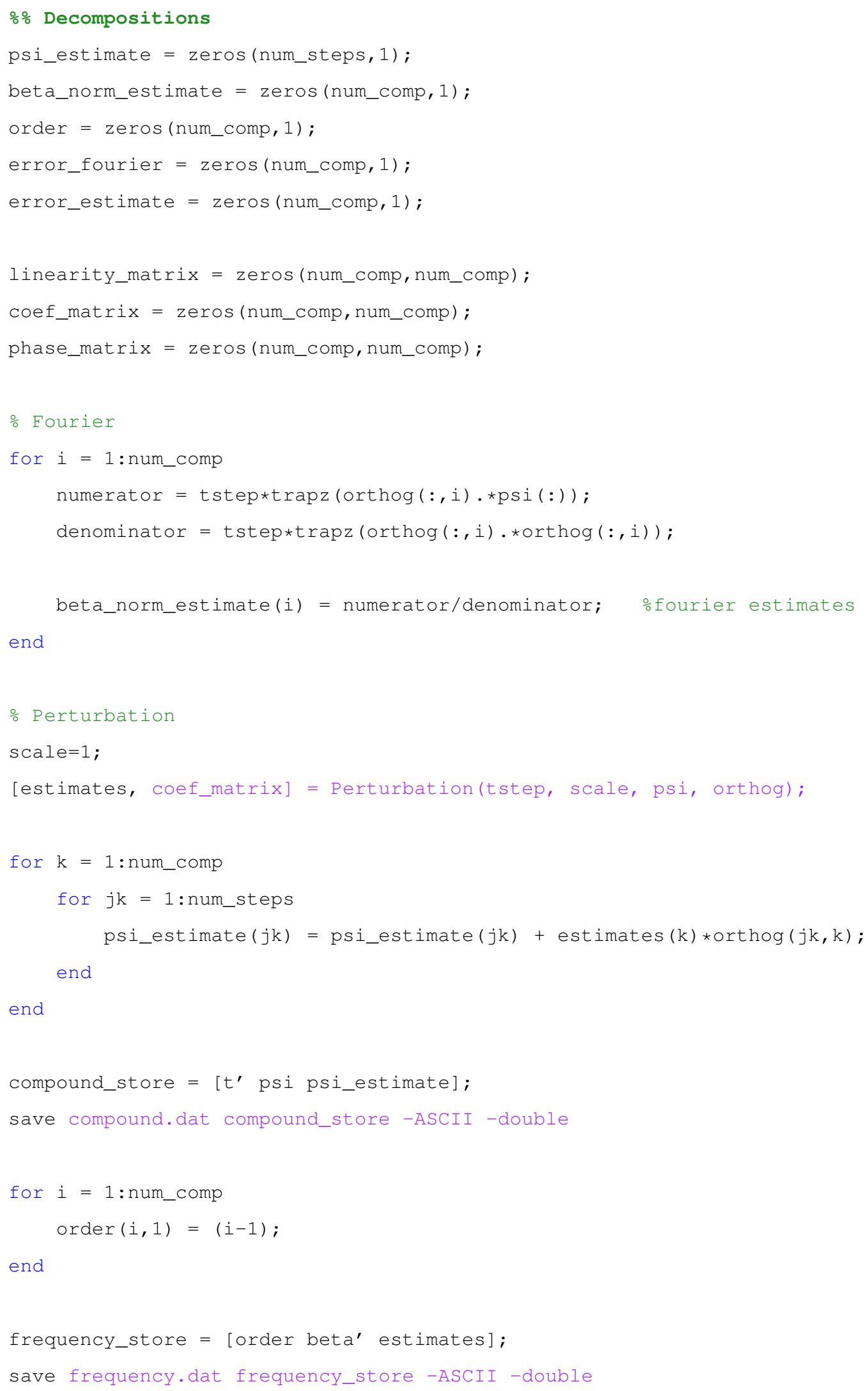




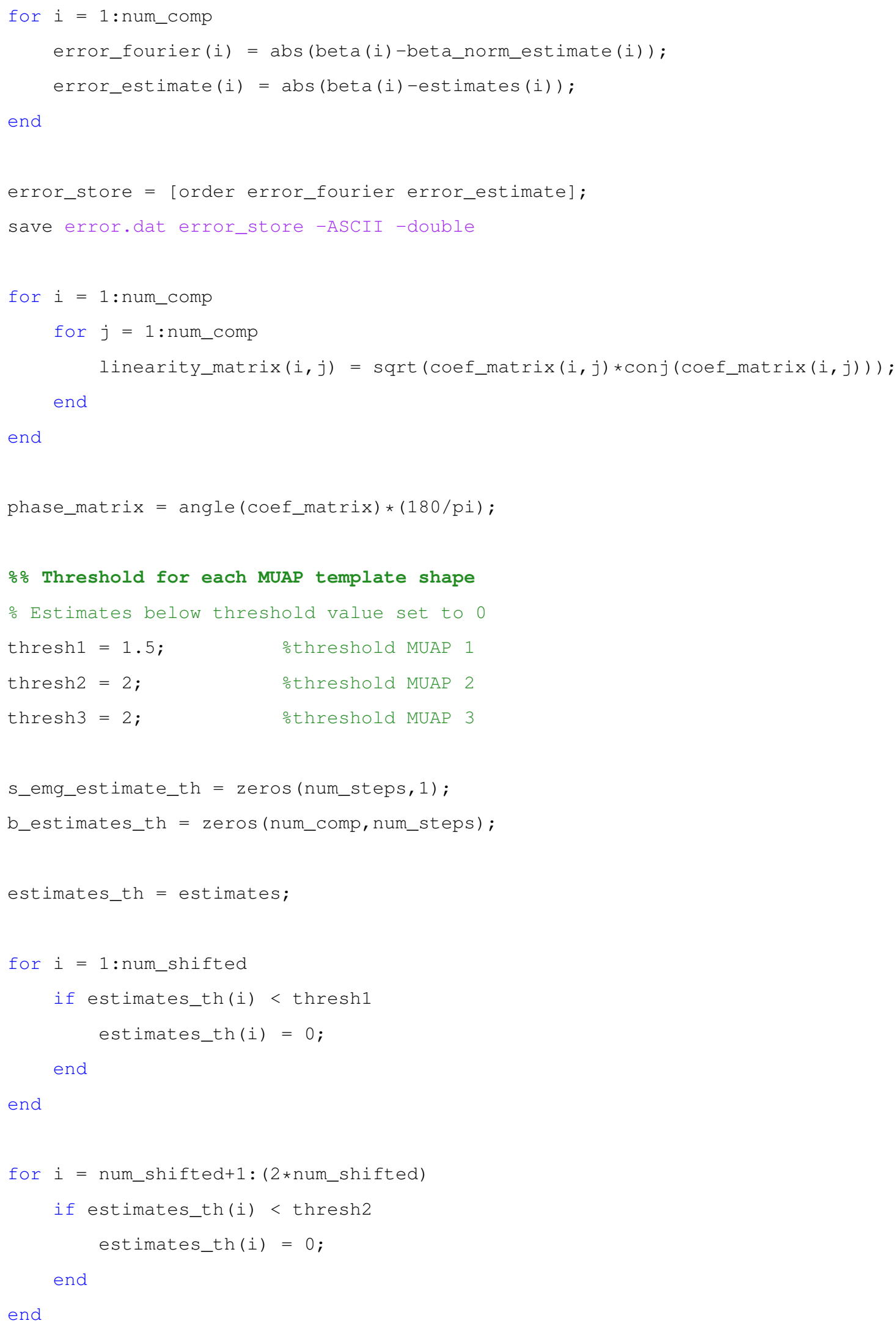




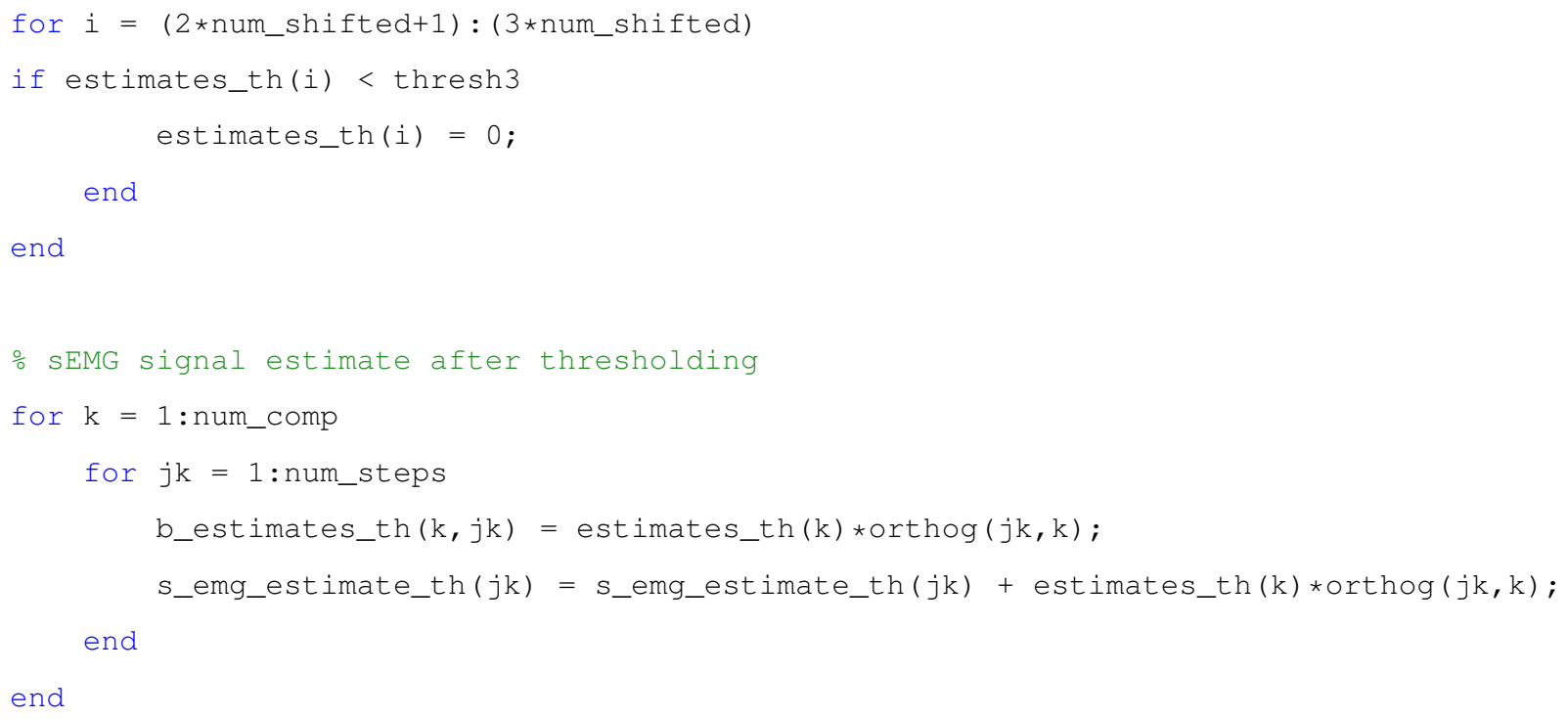




\section{Real sEMG MUAP Decomposition}

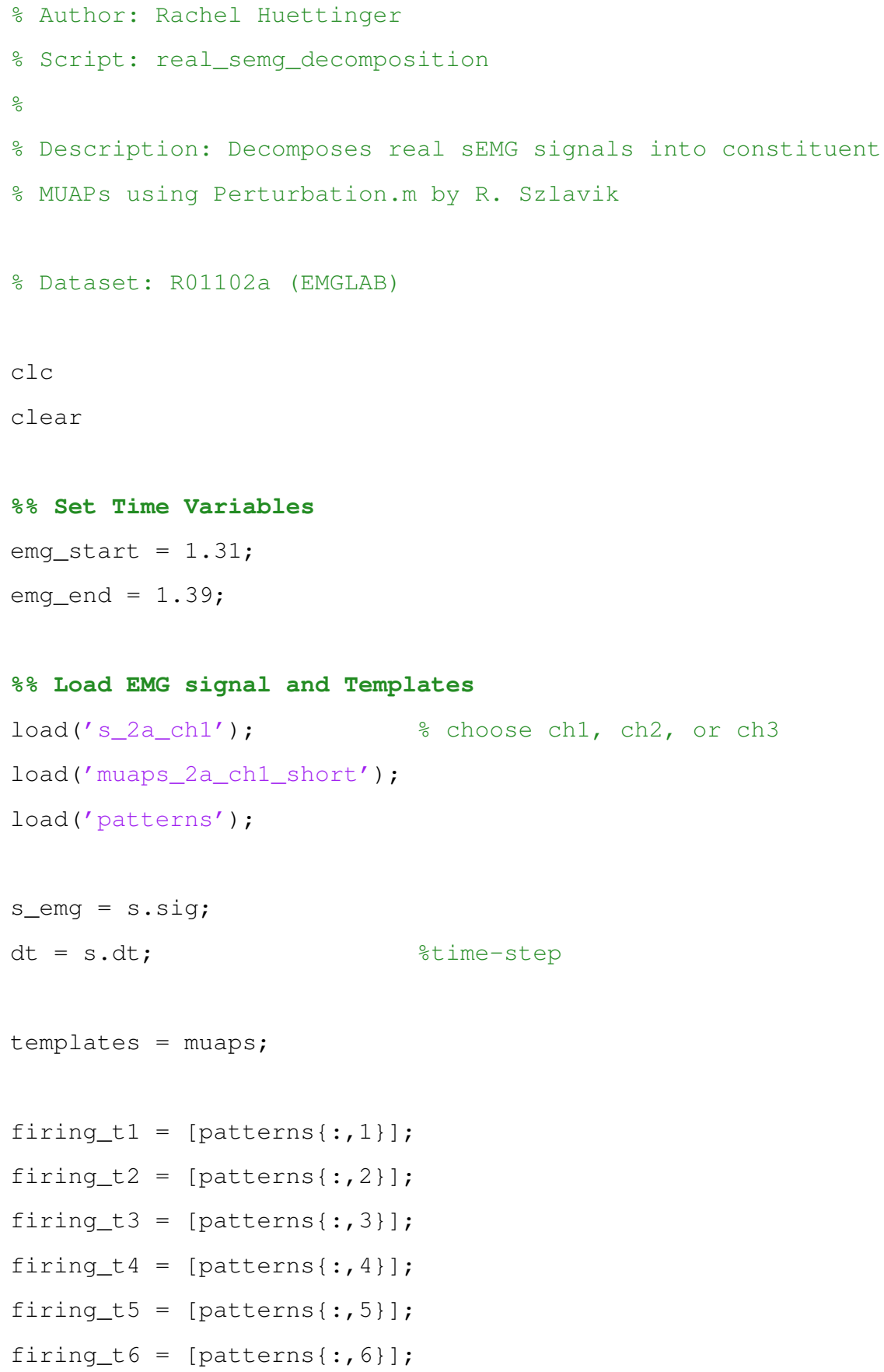




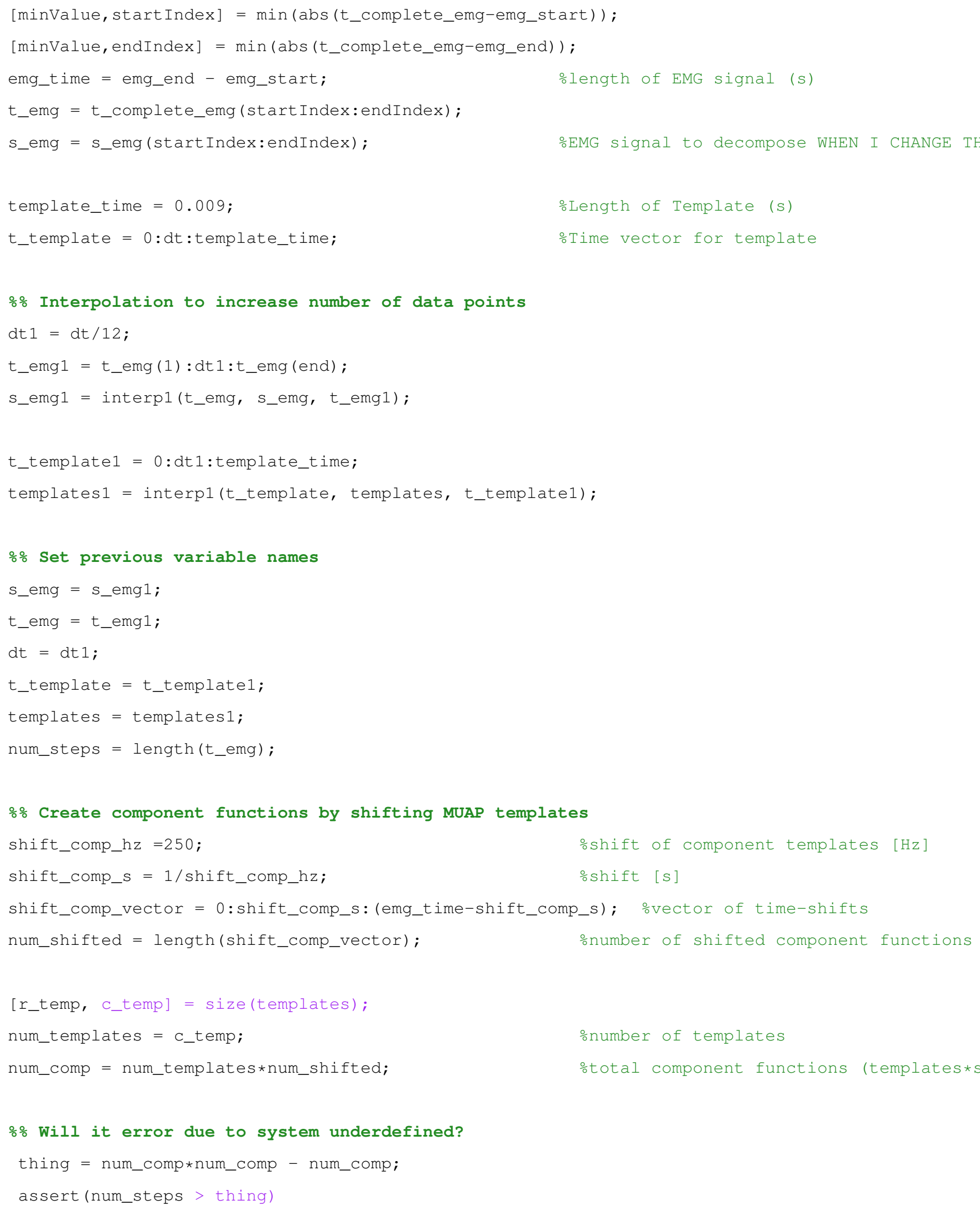




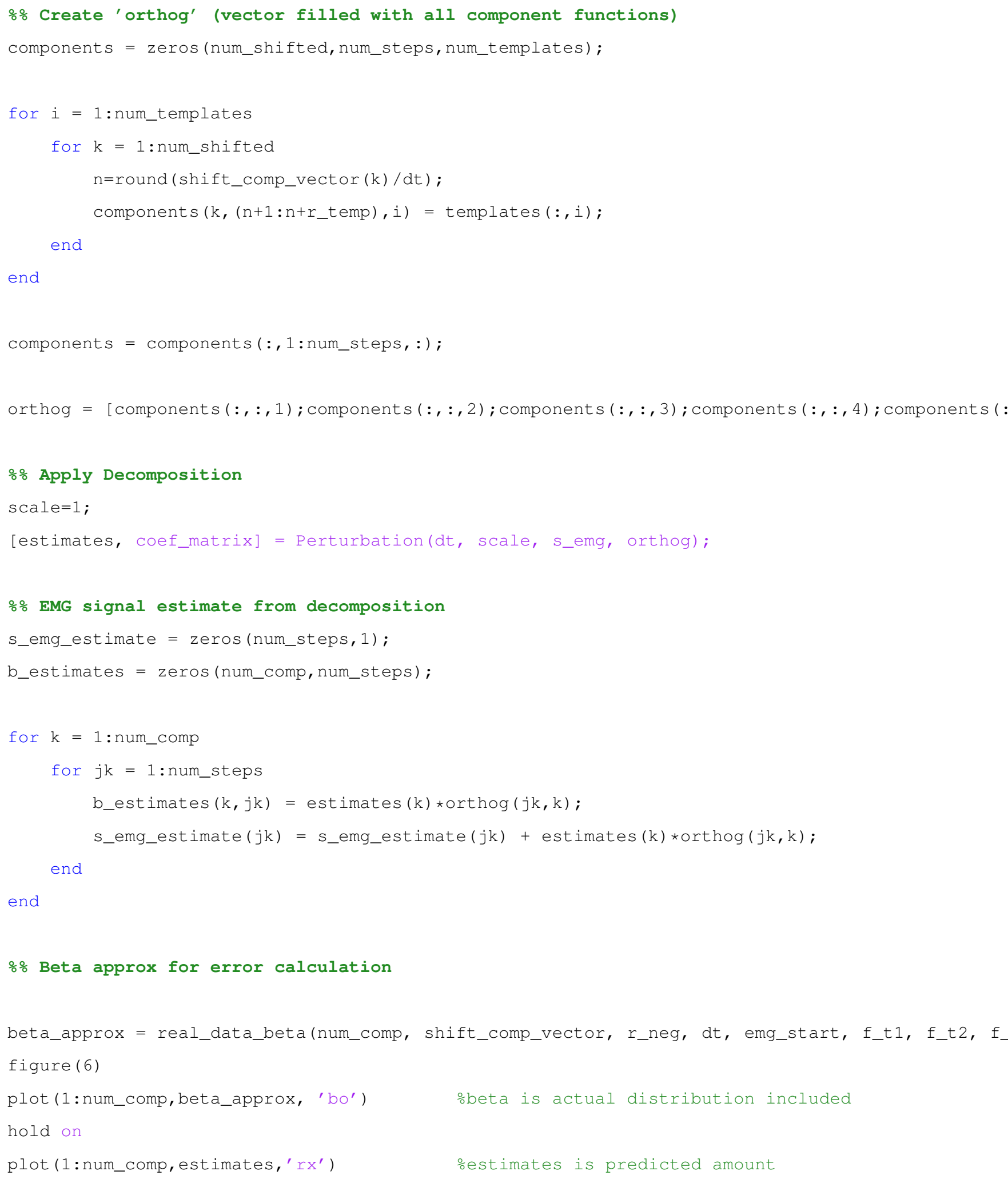

